\title{
Dynamic Underwater Glider Network for Environmental Field Estimation
}

\author{
R. Grasso, P. Braca, Member IEEE, S. Fortunati, Member IEEE, F. Gini, Fellow, IEEE, M. S. Greco, Fellow, IEEE
}

\begin{abstract}
A coordinated dynamic sensor network of autonomous underwater gliders to estimate 3D time-varying environmental fields is proposed and tested. Integration with a network of surface relay nodes and asynchronous consensus are used to distribute local information and achieve the global field estimate. Field spatial sparsity is considered and field samples are acquired by compressive sensing devices. Tests on simulated and real data demonstrate the feasibility of the approach with relative error performance within $10 \%$.
\end{abstract}

Index Terms-dynamic sensor networks, distributed processing, consensus protocols, compressive sensing, underwater autonomous gliders, environmental field estimation.

\section{INTRODUCTION}

$\mathrm{T}$ HE advent and improvement of autonomous underwater vehicle technology open wide new perspectives on sampling the ocean efficiently and persistently at feasible costs [1][2]. In particular, the autonomous capability of robotic sampling networks, able to satisfy in real time prescribed requirements, exhibits high quality ocean field estimation and forecast. At the same time, this kind of networks poses new challenging problems regarding the managing and the automatic control of such networks [3][4]. Recent advances in distributed statistical signal processing techniques related to the control and the inference in dynamic sensor networks, provide a robust theoretical framework to tackle several challenges [5][6][7][8][9]. Furthermore, this paradigm of autonomous network of vehicles permits the ocean sampling with a minimum amount of human supervision.

This work describes an approach for optimally estimating slowly varying environmental spatial fields in a distributed fashion by a fleet of autonomous underwater vehicles (agents), integrated with a network of relay nodes. The system architecture allows all the agents and all the relay nodes to converge to the optimal estimated field. These characteristics are suitable, in particular, for the control of a network of underwater vehicles, where each agent of the network can

This work has been funded by the NATO Allied Command Transformation (NATO-ACT) under the project ACT000405, Environmental Knowledge and Operational Effectiveness - Decisions in Uncertain Ocean Environments (EKOE-DUOE).

R. Grasso and P. Braca are with NATO STO CMRE, Viale San Bartolomeo 400, La Spezia, Italy (email: grasso@cmre.nato.int, braca@cmre.nato.int).

S. Fortunati, F. Gini and S. M. Greco are with the Dipartimento di Ingegneria dell'Informazione, University of Pisa, via G. Caruso 16, 56122 Pisa, Italy (e-mail: stefano.fortunati@iet.unipi.it, f.gini@iet.unipi.it, maria.greco@iet.unipi.it). communicate sporadically with one or more relay nodes (see Fig. 1).

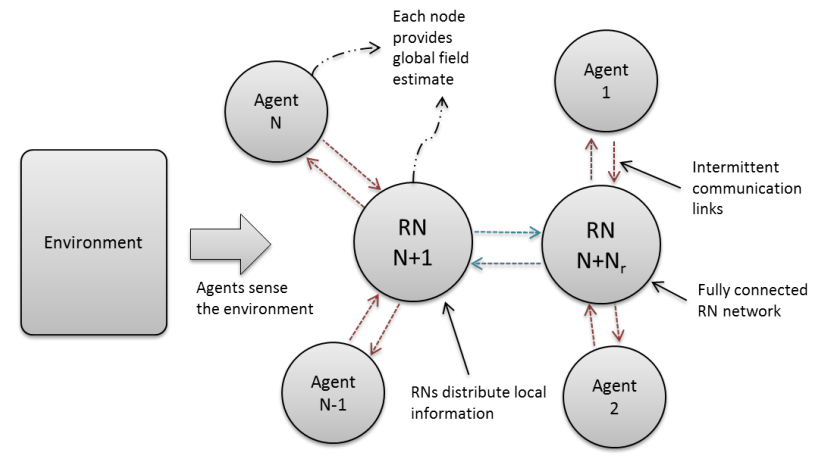

Fig. 1. Sensor network structure.

We focus on the case of an underwater network of agents such as gliders [1], that sporadically emerge to perform satellite or radio communications.

Underwater communications are not used as the glider agents considered in this work, mainly for technological limits (vehicle size and energy budget), are not equipped with an acoustic modem $(\mathrm{Tx} / \mathrm{Rx})$. The agents do not directly communicate each other, neither underwater nor at surface. Communications are only asynchronous at surface between agents and a network of relay nodes that is reachable by a vehicle with high probability through satellite/radio links when it is at surface. Synchronous communications at surface among agents are unfeasible as it is difficult to synchronize the surfacing phase of the vehicles. The sensor network envisioned in this work has a wide geographic extension to cover large areas of the order of tens/hundreds of $\mathrm{km}$. In order to exchange information frequently, directly between agents, by using underwater communications, the communication range should be sufficiently big in order to avoid the vehicles to communicate only when they are in proximity of each other. Considering the typical extension of the surveyed area and the slow speed of the vehicles (nominally $0.6 \mathrm{~m} / \mathrm{s}$ ) short communication ranges will make the information exchange very poor, degrading the overall performance of the system. Again, for the moment, a long distance underwater communication capability goes beyond the technological limits of the considered class of vehicles. Nevertheless, the distributed estimation algorithm and network control proposed in this work is still valid, with minor changes, in case technology allows for different communication architectures, such as an ad-hoc network of underwater nodes where gliders pass on information from one to another.

The relay node acts as an information gateway to asynchronously distribute the local information collected by a sensor to all the other sensors. The global estimation of the 
spatial field is, in this way, iteratively computed and somehow shared by all the nodes of the network. The estimated field can be retrieved by interrogating a node when this is reachable by the user. The information diffusion is based on the consensus protocol among sensors and the relay node(s) [7][10][11][12].

The relay nodes supporting the agent network of underwater gliders can include a single remote command and control centre communicating with gliders at surface through a satellite link (like Iridium) [1], oceanographic fixed surface buoys, surface vehicles (like wave gliders [13][14]) and/or an oceanographic mother ship all connected by satellite and/or radio-links or a combination of the previous options. Normally, these surface assets are deployed during oceanographic campaigns so that the additional cost of configuring them as a network of relay nodes is negligible. The spatial field of interest is assumed to be constant in time or slowly time-varying. Moreover, we also consider the case in which the field is spatially sparse, i.e. the field can be represented by a number of informative components that is lower than the total number of dictionary base functions used to represent it. The estimation algorithm is based on a sparsity aware Kalman filter (SA-KF) [15][16] to refine the solution by taking into account the field sparsity constraints. The sensors are equipped with a compressive sensing (CS) device [17] to compress the collected information directly at the sampling stage. In this way, the sensors can operate at a lower sampling rate than the original sensor rate preserving information and simplifying the sampling hardware on the side of the analog frontend [17].

The network sampling strategy is adaptive. In particular, the path of an agent of the network is optimized in such a way that the agents are forced to move into the most informative regions, e.g. see [9]. In other words, the measurements are collected in those areas where the estimate is more inaccurate.

The proposed architecture can be applied to map spatial fields for any measureable environmental parameter such as seawater temperature and optical properties, acoustic noise distribution and pollutant concentration. Moreover, with some suitable modifications, the system can become a target grid tracker as proposed in [15][16], or a distributed device for herding activity in intelligence and border security applications, as suggested in [19].

The architecture can find use especially in the case of largescale networks of autonomous underwater vehicles like gliders [1]. These vehicles typically perform underwater missions covering large areas and for long periods of time (even months). They can communicate to a command and control center through a satellite link (or a radio link in the proximity of the coast, mother ships and/or surface vehicles) only when at sea surface and cannot communicate underwater with other vehicles or gateways through an acoustic link at very long range, due to energy budget and communication equipment constraints. They form a multi-payload platform carrying on board several scientific sensors at the same time such as conductivity, temperature and depth (CTD) sensors, seawater optical parameter sensors and acoustic hydrophones, thus implying a great accumulation of data in the vehicle storage system.

This work follows the seminal papers [19] and [20] on spatial field distributed estimation by dynamic sensor networks in a centralized as well as de-centralized way for the static and dynamic cases. The protocols proposed in these works suppose that neighboring agents can communicate each other continuously. These papers do not take into account networks with intermittent communication links and the sparsity of the spatial field. Moreover, sensors work at the Nyquist rate and are not equipped with a CS device. The SAKF was introduced in [15][16], where the authors propose a grid tracking system taking advantage of the inherent sparsity of the surveillance scene (that is usually characterized by a number of targets that is much lower than the total number of grid cells). The work takes into account static sensors and no applications are considered to dynamic sensor networks.

The novel contribution of this paper consists in the design and the application of an adaptive dynamic network for 3D ocean field estimation in a distributed way by a fleet of underwater autonomous gliders. In particular, the originality of this work is in the combination of advanced well known techniques in sensor networks, distributed inference, dynamic network control and sparse sampling, and in their use in an oceanographic application that is challenging. The design solves the sporadic and asynchronous communication limiting factors, promotes a parsimonious and compact field representation by introducing spatial sparsity constraints, and directly compresses the acquired information at the sampling stage by using CS devices.

The paper aims at evaluating the performance of the system for specific scenarios. The scenarios here reported are based on simulated 3D static and dynamic spatially sparse fields and on a real non-sparse oceanographic forecast model of the sea water temperature, the Navy Coastal Ocean Model (NCOM) [18]. The achieved mean steady state relative error between the estimated and the true field is within $10 \%$.

This paper is organized as follows. Section 2 provides the overview of the system. In particular, the field decomposition is first introduced; the field estimation algorithm based on a centralized Kalman filter architecture is described and the CS sensor device model that is used to modify the original Kalman filter measurement equation is specified; the subsections on sparsity aware Kalman filter, agent control law and kinematic model of underwater autonomous glider close Section 2. In Section 3 the centralized architecture is distributed and the consensus protocol is detailed. Section 4 provides simulation results while Section 5 ends the paper drawing conclusions and highlighting future work.

\section{Centralized estimation}

This section provides an overview of the centralized field estimation algorithm. Methods to promote the sparsity in the estimation at the local nodes will also be introduced. The consensus protocol will be detailed later in Section 3. The estimation procedure relies on the expansion of the spatial field on a basis of known spatial functions, weighted by unknown coefficients which are in general time variant. The spatial field to be estimated can be written as [19]:

$$
g(\mathbf{r} ; t)=\sum_{j=1}^{L} c_{j}(t) \psi_{j}(\mathbf{r})=\Psi(\mathbf{r}) \mathbf{c}(t)
$$

where $\mathbf{r}$ is the spatial position vector in the region of interest 
(for instance $2 \mathrm{D}$ or $3 \mathrm{D}$, in a Cartesian reference system), $\mathbf{c}=\left[c_{1}, \ldots, c_{L}\right]^{T}, c_{j}$ is the $j$-th coefficient, $\psi_{j}(\mathbf{r})$ is the $j$-th base function and $L$ is the total number of base functions. For the sake of clarity, from now on, the time variable $t$ is dropped from the coefficient expressions. Given the base of spatial functions, $\Psi(\mathbf{r})=\left[\psi_{1}(\mathbf{r}), \ldots, \psi_{L}(\mathbf{r})\right]$, the problem of estimating the scalar field from the sensor measurements is equivalent to estimate the coefficient vector $\mathbf{c}$.

We assume here that the coefficient vector $\mathbf{c}$ is sparse, i.e. the coefficient vector has $K \ll L$ non-zero components whose amplitude and support are unknown. In this case, the vector $\mathbf{c}$ can be conveniently estimated using algorithms that take into account its sparsity. Here we use a sparsity aware Kalman filter, as suggested in [15][16]. Moreover, the network agents considered in this work are equipped with a sparse sensing acquisition device such as the random demodulator [17]. The coefficient vector can be estimated sequentially at a sampling rate lower than the Nyquist limit by including in the Kalman filter measurement equation the sparse device model.

The following subsections will detail the estimation algorithm in various forms. In particular, the centralized model is first introduced, together with the coordinated network control. The CS version of the algorithm is described introducing first the random demodulator measurement equation and then the sparsity aware Kalman filter. A description of the agent used in simulation tests is also provided, including the kinematic and the operational and communication constraints.

\section{A. Centralized estimation}

Centralized coefficient estimation is based on a network of agents that communicate to a fusion center (FC) their local field estimates at each time step $k$. The FC processes the local estimates by averaging them to obtain the global field estimate. The centralized algorithm here detailed is not feasible for a glider network because it would require local estimates available at the fusion center at each time step. This is not possible as the vehicles cannot communicate acoustically underwater with the FC, but only when at surface, typically every 1 to 3 hours, by a satellite/radio link. The centralized system is then an ideal system that is used to compare the consensus based distributed solution detailed in section III.

The estimation-level fusion (similarly to track-to-track fusion as in [21][22]), rather than the Kalman filter centralized solution that fuses the sensor measurements [19], has been chosen for several reasons. The fusion algorithm here used is optimum in the minimum mean square error (MMSE) and maximum likelihood (ML) sense considering the sensors estimates as independent [21][22][23]. As this hypothesis is not always true, the FC processor is in general sub-optimal, but with the advantage of reducing complexity. The FC (as well as the RNs in the consensus algorithm as detailed in section III) does not explicitly promotes sparsity. Instead, the sparsity is promoted at the agent local level (see section II.B and II.C) and the sensor estimates are fused in the FC (or in the RNs) similarly to [24] in which a pool of sparse solutions are averaged in order to improve the final estimate. The fused estimate is then feedback to the sensors to reinitialize the local estimates. The sensors indirectly share information among them and, because the agents share the same field model, the local estimates converge in terms of support and amplitude to the true global state vector after a transitory phase. Moreover, being the distributed consensus algorithm based on the fusion of local agent estimates as detailed in section III, the comparison with the centralized solution is more consistent and straightforward. The comparison with a measurementlevel centralized solution is also possible and it can be a further topic for future work.

Compared to a solution in which the agents exchange their measurements with the FC (or the RN nodes) and the control of the agent network is centralized as in [19], the approach here followed, based on estimation-level fusion and local agent control, has a higher communication overhead, but makes the integration of new agents in the network more flexible as a measurement model is not required at the $\mathrm{FC}$, and the FC has not to know the local agent measurement model and the network spatial configuration at a given time step (the sensor positions are not needed at the FC). Moreover, the local control, that is based on the agent position, the measurement model and the estimation covariance (as detailed in section II.D), is more robust to communication failures [25] as an agent can apply control using local estimates instead of fused estimates at the $\mathrm{FC}$ which are not available due to a missed communication [14].

The local agent sequential estimation is performed by a Kalman filter in which the coefficient dynamic is modeled by a linear state space equation shared by all the agents:

$\mathbf{c}_{i, k}=\mathbf{F}_{k} \mathbf{c}_{i, k-1}+\mathbf{G}_{k} \mathbf{u}_{k}+\mathbf{n}_{k}$,

where $\mathbf{F}_{k}$ is the state transition matrix, $\mathbf{u}_{k}$ is a $P$ dimensional column vector of exogenous forcing factor, weighted by the known $L \times P$ matrix $\mathbf{G}_{k}$, and $\mathbf{n}_{k}$ are Gaussian distributed independent noise vectors with known covariance matrix $\mathbf{Q}_{k}=\operatorname{diag}\left(\left[\sigma_{1, k}^{2}, \ldots, \sigma_{L, k}^{2}\right]^{T}\right)$, where $\sigma_{j, k}^{2}$ is the variance of the $j$-th coefficient, with $j=1, \ldots, L$.

Assuming a network composed of $N$ sensors, the $i$-th sensor, for $i=1, \ldots, N$, acquires at each time step a noisy measurement $y_{i, k}$ of the field. The $i$-th sensor measurement equation can be expressed as follows:

$y_{i, k}=\mathbf{h}_{i, k} \mathbf{c}_{i, k}+e_{i, k}$,

where $\mathbf{h}_{i, k}=\left[\psi_{1}\left(\mathbf{r}_{i, k}\right), \ldots, \psi_{L}\left(\mathbf{r}_{i, k}\right)\right]$ is the measurement vector and $\mathbf{r}_{i, k}$ is the position of the $i$-th sensor at time step $k$. The scalar $e_{i, k}$ is a Gaussian uncorrelated random noise, independent from $\mathbf{n}_{k}$, with variance $[\mathbf{R}]_{i, k}=\rho_{i, k}^{2}$. Each sensor runs the Kalman filter prediction and update steps to provide the sequential estimate of the coefficient vector $\hat{\mathbf{c}}_{i, k}$ and its 
covariance matrix $\hat{\mathbf{C}}_{i, k}$ to the FC. The FC fuses the local information matrix, $\hat{\mathbf{D}}_{i, k}=\hat{\mathbf{C}}_{i, k}^{-1}$, and the local information vector $\hat{\mathbf{g}}_{i, k}=\hat{\mathbf{D}}_{i, k} \hat{\mathbf{c}}_{i, k}$ by a weighted sum:

$$
\begin{aligned}
& \hat{\mathbf{g}}_{k}=\sum_{i=1}^{N} w_{i} \hat{\mathbf{g}}_{i, k}, \\
& \hat{\mathbf{D}}_{k}=\sum_{i=1}^{N} w_{i} \hat{\mathbf{D}}_{i, k},
\end{aligned}
$$

and then retrieves the global coefficient vector as $\hat{\mathbf{c}}_{k}=\hat{\mathbf{D}}_{k}^{-1} \hat{\mathbf{g}}_{k}$ and its covariance matrix as $\hat{\mathbf{C}}_{k}=\hat{\mathbf{D}}_{k}^{-1}$. The FC broadcasts the global estimates to the sensors to update the local estimates with the global ones.

In principle, the dynamic equation as well as the measurement equation can be non-linear. In this case, a nonlinear sequential filter like the unscented Kalman filter (UKF) [26] can be used. In this work, only the linear case is considered. When the dynamic of the coefficients is unknown, equation (2) is used with $\mathbf{F}_{k}=\mathbf{I}_{L}$ and $\mathbf{G}_{k}=\mathbf{O}_{L \times P}$ ( $\mathbf{I}_{L}$ is the $L \times L$ identity matrix and $\mathbf{O}_{L \times P}$ is the $L \times P$ matrix having all zero entries), and with $\sigma_{j, k}^{2}$ as free parameters that can be tuned to adjust the velocity at which the system adapts its estimate to the true dynamic of the coefficients [27]. The tradeoff to be considered is between the filtering response of the system and the estimate residual error [27].

\section{B. Sensor model and CS measurement equation}

Compressive sensing provides both the theoretical framework and the practical tools to efficiently approach the sampling and the reconstruction of sparse signals [28][29]. The CS sampling process is modeled by a linear system as follows:

$$
\mathbf{y}=\Phi \mathbf{x}
$$

where $\mathbf{x}=\left[x_{1}, \ldots, x_{W}\right]^{T}$ is a sparse vector of $W$ samples acquired at the Nyquist rate, $\Phi$ is an $M \times W$ sampling matrix, with $M<W$, and $\mathbf{y}=\left[y_{1}, \ldots, y_{M}\right]^{T}$ is a vector of $M \mathrm{CS}$ measurements. If the vector $\mathbf{x}$ has $K$ unknown components different from zero, with $K<<W$, CS theory states that x can be exactly recovered by minimizing its $L_{1}$-norm constrained by (6) from a number of CS measurements $\mathrm{M}$ proportional to $K \ln (W)$. CS theory provides a way to sample more efficiently sparse signals, where the sparsity is in a given linear transformed domain. This is typically carried out by developing cheap analog sampling devices working at a lower sampling rate. A large part of research efforts in CS is devoted to the implementation of sampling schemes that can be modeled by (6) with random sampling matrices.

The random demodulator (RD) device, proposed in [17] (see Fig. 2), consists in first modulating the analog input signal, $x(t)$, with an analog random sequence, $p_{c}(t)$, of impulses at Nyquist rate (the chip sequence) with amplitude that takes \pm 1 equiprobable values. The modulator is followed by an integrator and a sample and hold device that works at a lower sampling rate than Nyquist.

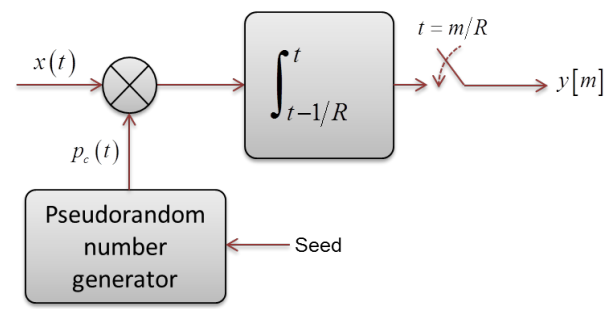

Fig. 2. Random demodulator scheme. Adapted from Tropp et al [17].

If the observation time is normalized to 1 , the Nyquist rate is $1 / W$, the integration is performed in the interval $[t, t-1 / M)$ and the sample and hold device works at a rate equal to $1 / M$. At the end of the observation time, the device provides a vector of $M$ samples given by (6). The sampling matrix $\Phi$ that models the RD in (6) can be decomposed as the product of two matrices, $\Phi=\mathbf{H} \Lambda$, where

$$
\mathbf{H}=\left[\begin{array}{ccccccccccc}
\frac{W / M}{1} & 1 & 1 & 0 & 0 & 0 & 0 & \ldots & 0 & 0 & 0 \\
0 & 0 & 0 & 1 & 1 & 1 & 0 & \ldots & 0 & 0 & 0 \\
0 & 0 & 0 & \ldots & & & \ldots & 0 & 1 & 1 & 1
\end{array}\right]
$$

is a $M \times W$ matrix simulating the integration and $\Lambda=\operatorname{diag}\left(\left[p_{0}, \ldots, p_{W-1}\right]^{T}\right)$, with $p_{j}= \pm 1$, a sequence of equiprobable binary symbols simulating the chip sequence. Each row of $\mathbf{H}$ contains a sequence of 1's with a length of $B=W / M$ samples starting at the $(m B+1)$-th column, with $m=0, \ldots, M-1$.

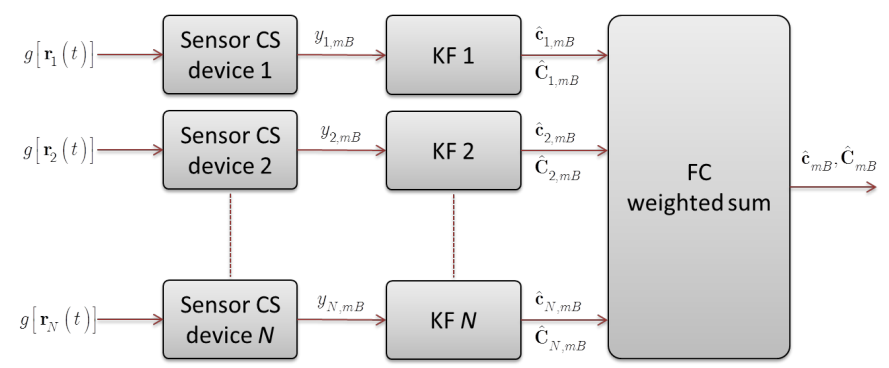

Fig. 3. Centralized Kalman filter estimation from CS samples of the scalar field.

In this work, each sensor is equipped with a CS device like the RD. The local Kalman filter directly processes the CS samples working at a lower than Nyquist sampling rate (see Fig. 3). In order to allow the Kalman filter to process such samples, the measurement equation (3) has to be modified to properly model the RD sampling process. If $B$ is the CS block of Nyquist samples that are weighted by the chip sequence and then averaged, the CS sample at time $k=m B$ of the $i$-th sensor is: 
$y_{i, m B}=\frac{1}{B} \mathbf{p}_{i, m B}^{T} \mathbf{H}_{i, m B} \mathbf{c}_{m B}+\frac{1}{B} \mathbf{p}_{i, m B}^{T} \mathbf{e}_{i, m B}$,

where

$$
\mathbf{H}_{i, m B}=\left[\mathbf{h}_{i, m B-B+1}^{T}, \ldots, \mathbf{h}_{i, m B-1}^{T} \mathbf{h}_{i, m B}^{T}\right]^{T}
$$

and

$$
\mathbf{h}_{i, m B-b}=\left[\psi_{1}\left(\mathbf{r}_{i, m B-b}\right), \ldots, \psi_{L}\left(\mathbf{r}_{i, m B-b}\right)\right]
$$

With $b=B-1, \ldots, 0 . \mathbf{p}_{i, m B}=\left[p_{i, m B, 1}, \ldots, p_{i, m B, B}\right]^{T}$ is the chip random sequence of the $i$-th sensor and $\mathbf{e}_{i, m B}=\left[e_{i, m B-B+1}, \ldots, e_{i, m B}\right]^{T}$ is the noise sequence in (3), for $k=m B-B+1, \ldots, m B$. The local Kalman filter predicts the measurement using (8) and updates the coefficient estimate prediction, $\hat{\mathbf{c}}_{i, m B \mid(m-1) B}=\mathbf{F}_{m B} \hat{\mathbf{c}}_{i,(m-1) B}+\mathbf{G}_{m B} \mathbf{u}_{m B}$, by using the innovation between the actual measurement and the predicted one. The final measurement equation is:

$y_{i, m B}=\mathbf{q}_{i, m B} \mathbf{c}_{i, m B}+\varepsilon_{i, m B}$,

where

$\mathbf{q}_{i, m B}=\frac{1}{B} \mathbf{p}_{i, m B}^{T} \mathbf{H}_{i, m B}$

and $\varepsilon_{i, m B}=\mathbf{p}_{i, m B}^{T} \mathbf{e}_{i, m B} / B$, is a Gaussian random noise having the same variance of $e_{i, k}$ (this is due to the particular choice of the chip sequence).

\section{Sparsity aware Kalman filter}

In order to take advantage of the sparse structure of the coefficient vector, the local Kalman filter applies a further step after the measurement update that refines the coefficient estimation enforcing sparsity (see Fig. 4). The filter is inspired by the so called sparsity aware Kalman filter (SA-KF) as proposed in [15][16]. This filter substitutes the classical Kalman update step with a gradient descent iterative algorithm initialized by the predicted state vector estimate in order to minimize a three term functional that include the $\mathrm{L}_{1}$-norm of the state vector. The approach followed here is similar to [15][16] with the iterative minimization initialized by the state vector estimate updated by the classical Kalman update step. In particular, the refinement step consists in minimizing a cost function composed of three additive terms: (i) the square error between the coefficient $\mathbf{c}_{i, m B}$ and the Kalman estimate $\hat{\mathbf{c}}_{i, m B}$, weighted by $\hat{\mathbf{C}}_{i, m B}^{-1}$; (ii) the square error between the measurement vector $y_{i, m B}$ and the predicted measurement $\mathbf{q}_{i, m B} \mathbf{c}_{i, m B}$; (iii) the $\mathrm{L}_{1}$-norm of the coefficient vector, that is the term that promotes sparsity in the solution, weighted by a constant parameter $\lambda_{S A}$ that controls the sparsity-bias tradeoff [15][16].

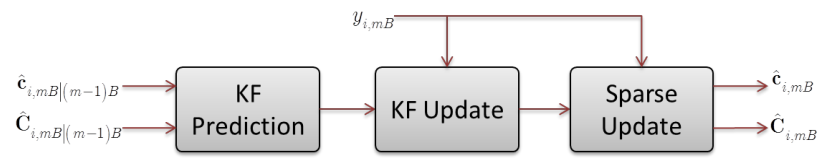

Fig. 4. Kalman filter with the sparse refining scheme.

The refined estimate of the coefficient vector is obtained by minimizing the following cost function:

$$
\begin{aligned}
\tilde{\mathbf{c}}_{i, m B} & =\arg \min \left[J_{\mathbf{c}_{m B}}\left(\mathbf{c}_{i, m B}\right)\right] \\
& =\arg \min _{\mathbf{c}_{m B}}\left[\left\|\mathbf{c}_{i, m B}-\hat{\mathbf{c}}_{i, m B}\right\|_{\hat{\mathbf{C}}_{i, m B}^{-1}}^{2}+\right. \\
& \left.+\left\|y_{i, m B}-\mathbf{q}_{i, m B} \mathbf{c}_{i, m B}\right\|_{R_{i, m B}^{-1}}^{2}+\lambda_{S A}\left\|\mathbf{c}_{i, m B}\right\|_{1}\right] .
\end{aligned}
$$

Equation (13) is solved iteratively by a stochastic gradient descent algorithm. Given the coefficient state transition equation (2) with $\mathbf{F}_{k}=\mathbf{I}_{L}$ and $\mathbf{G}_{k}=\mathbf{O}_{L \times P}$ and the measurement matrix (12), the gradient of the cost function in (13) can be written as:

$$
\begin{aligned}
\nabla J_{S A}\left(\mathbf{c}_{i, m B}\right)= & 2\left[-\hat{\mathbf{C}}_{i, m B}^{-1}\left(\mathbf{c}_{i, m B}-\hat{\mathbf{c}}_{i, m B}\right)-\right. \\
& \left.-\mathbf{q}_{i, m B}^{T} R_{i, m B}^{-1}\left(y_{i, m B}-\mathbf{q}_{i, m B} \mathbf{c}_{i, m B}\right)+\lambda_{S A} \mathbf{1}_{L}\right]
\end{aligned}
$$

that is valid for positive coefficients for which the $L_{1}$-norm is differentiable.

The refined solution is found by iterating the following equation:

$\tilde{\mathbf{c}}_{i, m B}(l+1)=\tilde{\mathbf{c}}_{i, m B}(l)-\gamma_{S A} \nabla J\left[\tilde{\mathbf{c}}_{i, m B}(l)\right]$,

where $\gamma_{S A}$ is an update step size parameter, until the error between $\tilde{\mathbf{c}}_{m B}(l+1)$ and $\tilde{\mathbf{c}}_{m B}(l)$ is below a given threshold or after a maximum number of iterations. The iteration starts with the coefficient estimate at the output of the regular Kalman filter stage i.e. $\tilde{\mathbf{c}}_{i, m B}(0)=\hat{\mathbf{c}}_{i, m B}$. The estimate can also be constrained; in case of non-negativity constraints, for instance, the estimate (15) can be projected onto the nonnegative orthant as proposed in [15][16].

In this work, a modification to the algorithm (13) is proposed, in which the $L_{1}$-norm term is substituted by a smoothed approximation of the $L_{0}$-norm of the coefficient vector [30]:

$$
\begin{aligned}
F_{\zeta_{S A}}\left(\mathbf{c}_{i, m B}\right) & =L-\sum_{j=1}^{L} \exp \left(-c_{i, j, m B}^{2} / 2 \zeta_{S A}^{2}\right) \\
& =L-\sum_{j=1}^{L} f_{\zeta_{S A}}\left(c_{i, j, m B}\right)
\end{aligned},
$$


referred to as smoothed $L_{0}$-norm ( $\mathrm{SL}_{0}$-norm). The limit of (16) as $\zeta_{S A}$ approaches zero is the $L_{0}$-norm of the coefficient vector i.e.:

$$
\lim _{\zeta_{S A} \rightarrow 0} F_{\zeta_{S A}}\left(\mathbf{c}_{i, m B}\right)=\left\|\mathbf{c}_{i, m B}\right\|_{0}
$$

The $\mathrm{SL}_{0}$-norm is a better approximation of the $L_{0}$-norm and it is differentiable everywhere. Equation (16) can be included in the objective function (13) with a small $\zeta_{S A}$, instead of the $L_{1}$-norm, and the resulting objective function can be minimized by using the gradient algorithm (15), with the gradient vector that is equal to:

$$
\begin{gathered}
\nabla F_{\zeta_{S A}}\left(\mathbf{c}_{i, m B}\right)= \\
\zeta_{S A}^{2}\left[c_{i, 1, m B} f_{\zeta_{S A}}\left(c_{i, 1, m B}\right), \cdots\right. \\
\left.\cdots, c_{i, L, m B} f_{\zeta_{S A}}\left(c_{i, L, m B}\right)\right]^{T}
\end{gathered}
$$

The final estimate is then updated with the refined solution, i.e. $\hat{\mathbf{c}}_{i, m B}=\tilde{\mathbf{c}}_{i, m B}$, accepting the approximation of considering the covariance of the final estimate equal to the covariance at the output of the regular Kalman filter.

Several approaches to enforce sparsity in the Kalman filter have been proposed in the literature other than [15][16], such as the one based on pseudo measurement-norms and quasinorms as described in [31]. The result section reports a comparison among the refining procedure here proposed, the classical SA-KF in [15][16] and the pseudo measurement approach in [31], showing that the first approach, outperforms the others using the same number of iterations.

\section{Network control}

Following [19], the local sensor control is given by updating the agent position in order to minimize the average covariance of the scalar field estimate at the time step $k+1$ with respect to the agent position. The field covariance is given by:

$$
J=\int_{A} \Psi(\mathbf{r}) \hat{\mathbf{C}}_{i, k+1} \Psi^{T}(\mathbf{r}) d A
$$

where the integration is over the whole area of interest, with $A \in \mathbb{R}^{2}$ or $A \in \mathbb{R}^{3}$, in which the network of agents is constrained to operate. As in [19], the dynamical model of the $i$-th agent is

$$
\mathbf{r}_{i, k+1}=\mathbf{r}_{i, k}+\mathbf{f}_{i, k}
$$

where the control input $\mathbf{f}_{i, k}$ is implemented by a gradient control law as follows:

$$
\mathbf{f}_{i, k}=-\left.S \frac{\partial J}{\partial \mathbf{r}_{i}}\right|_{\mathbf{r}_{i}=\mathbf{r}_{i, k}}
$$

where

$$
\frac{\partial J}{\partial \mathbf{r}_{i}}=\int_{A} \Psi(\mathbf{r}) \frac{\partial \hat{\mathbf{C}}_{i, k+1}}{\partial \mathbf{r}_{i}} \Psi^{T}(\mathbf{r}) d A
$$

and $S$ is a constant gain.

According to [19] the $n$-th component of the control input vector, with $n=1,2,3$ in the $3 \mathrm{D}$ case, is given by an expression involving the state covariance matrix, the measurement matrix (10) and its gradient with respect to the agent position:

$f_{i, n}=2 S R_{i}^{-1} \int_{A} \Psi(\mathbf{r}) \hat{\mathbf{C}}_{i} \frac{\partial \mathbf{h}_{i}^{T}}{\partial r_{i, n}}\left(\mathbf{r}_{i}\right) \mathbf{h}_{i}\left(\mathbf{r}_{i}\right) \hat{\mathbf{C}}_{i} \Psi^{T}(\mathbf{r}) d A$

where $\mathbf{h}_{i}\left(\mathbf{r}_{i}\right)$ is the agent measurement matrix (10) in the noncompressed domain, $\mathbf{r}_{i}=\left[r_{i, 1}, r_{i, 2}, r_{i, 3}\right]$ and $R_{i}=\rho_{i}^{2}$. The time step index has been dropped for the sake of clarity. As showed in (23), the control law depends on the agent position through the measurement matrix. The derivatives of the measurement matrix with respect to $r_{i, n}$ :

$$
\frac{\partial \mathbf{h}_{i}}{\partial r_{i, n}}=\left[\frac{\partial \psi_{1}\left(\mathbf{r}_{i}\right)}{\partial r_{i, n}}, \ldots, \frac{\partial \psi_{L}\left(\mathbf{r}_{i}\right)}{\partial r_{i, n}}\right]
$$

in case $\psi_{j}\left(\mathbf{r}_{i}\right)$ (with $\left.j=1, \ldots, L\right)$ is a Gaussian radial basis function (RBF) with given mean vector $\overline{\mathbf{r}}_{j}=\left[r_{j, 1}, r_{j, 2}, r_{j, 3}\right]$ and spreading parameter $\beta_{j}$, are given by:

$\frac{\partial \psi_{j}\left(\mathbf{r}_{i}\right)}{\partial r_{i, n}}=-2 \frac{r_{i, n}-\bar{r}_{j, n}}{\beta^{2}} \psi_{j}\left(\mathbf{r}_{i}\right)$,

with $\psi_{j}\left(\mathbf{r}_{i}\right)=\exp \left(-\left\|\mathbf{r}_{i}-\overline{\mathbf{r}}_{j}\right\|^{2} / \beta^{2}\right)$.

In the ideal centralized system, the control law can be applied by the $i$-th agent at each time step $k$. The trajectory followed by the agent is the one that would allow it to optimally collect non-compressed measurements along the way. An approximation can be used by applying the control law at each $k=m B$ so that the first position within the next CS sampling window, at $k=m B+1$, is the optimal one. This is the method used in the simulations to compare the centralized and the distributed consensus solutions as reported in the results section. In both cases, the Kalman prediction of the covariance matrix, $\hat{\mathbf{C}}_{i, k+1 \mid k}$, is actually used in (23) to calculate the control vector. In the case of a glider agent, in the consensus based distributed system, the control is applied when the vehicle is at surface. The dynamic of the agents (20) is adapted, as specified in the subsection below, to model the behavior of an underwater glider vehicle, having a constant speed (in absence of sea current), a constrained vertical plane dynamic and a waypoint guidance system. The control law in this case is used 
to optimally steer the direction of the vehicle on the horizontal plane toward the new waypoint.

\section{E. Agent kinematic model: the underwater glider}

In this work, underwater autonomous gliders [1] are considered to test the network architecture on simulated scenarios.

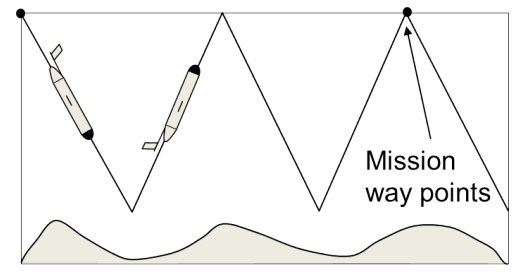

a)

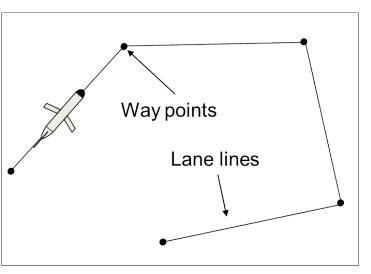

b)
Fig. 5. Glider mission plan: a) way points and lane lines in the horizontal plane, and b) yo-yo trajectory in the vertical plane.

Normally, in a typical mission setup, a glider moves through a $3 \mathrm{D}$ space following a saw tooth shape trajectory in the vertical plane and a waypoint list in the horizontal plane as in Fig. 5 to acquire measurements along the water column. The vehicle can be programmed to surface at each waypoint (typically every 1 to $3 \mathrm{~h}$ ). The trajectory is composed of a certain number of dive/climb cycles in the interval between two waypoints. The data, collected along the water column, are stored and finally transmitted when at surface to a control room, to be used later, for example, in assimilation algorithms for ocean forecasting.

The glider dynamic model considered in this work assumes a constant velocity without water current disturbances (effects of water current will the subject of future investigations), constrained to follow a yo-yo trajectory in the vertical plane with given climbing and diving target depths [32]. The glider, in absence of currents, navigates in the vertical plane along a yo-yo segment with a constant pitch angle $\phi$. The control vector (21) is normalized and multiplied by the total glider speed $V$ to take into account the constant speed constraint:

$\tilde{\mathbf{f}}_{i, m B}=V \mathbf{f}_{i, m B} /\left\|\mathbf{f}_{i, m B}\right\|$

Because the vehicle pitch is maintained constant during a dive/climb phase, the 2D horizontal plane components of (26) are used in the control law, at each glider surfacing when a waypoint is reached, to sub-optimally steer the vehicle direction toward the new waypoint [14] be reached after a given mean time, typically from 1 to $3 \mathrm{~h}$.

\section{DISTRIBUTED CONSENSUS ALGORITHM}

In this section, the centralized model of Section II is modified to overcome the physical operational constraints of a typical underwater glider vehicle and to allow the deployment and the automatic control of a network of such vehicles in a decentralized way. The resulting network architecture has a switching topology [33] and is based on the consensus paradigm [34][35] in which the information is diffused among the agents through the relay nodes, which act as an information gateway. Fig. 6 shows the basic structure of a single agent and a relay node $(\mathrm{RN})$ and the information flow between them.

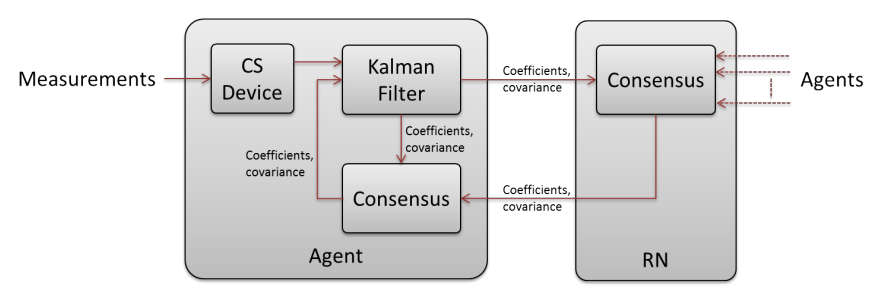

Fig. 6. Agent and RN structure.

\section{A. Consensus protocol}

The following assumptions are made for the proposed network model. Agents can communicate with a relay node at random instants. Agents cannot communicate among each other. Each agent sequentially estimates the coefficient vector by means of a SA-KF from local field measurements acquired by a CS device such as the RD (see details in Section II).

Each agent updates its position applying the control law and using the local prediction of the coefficient estimate covariance matrix. Agents communicating with the relay nodes transmit their local coefficient vector estimate and covariance, no field measurements are provided to relay nodes. Relay nodes distribute their estimate (coefficient vector estimate and covariance) to the connected agents and relay node update their coefficient vector estimate and covariance by combining the local agent's estimates, when available, through the average consensus algorithm [10][11], or propagates the previous estimate if no agents are connected. Agents connected to relay nodes update their local estimates (coefficient vector and covariance) by using relay node estimates through average consensus.

The protocol allows the global information to "intermittently flow" into the network through relay nodes with a collaborative behavior among the agents who emerge above the sea surface to start the communication. Realistic numerical simulations show that all local agent estimates and relay node estimates statistically converge close to the true global coefficient vector (i.e. the network reaches a consensus).

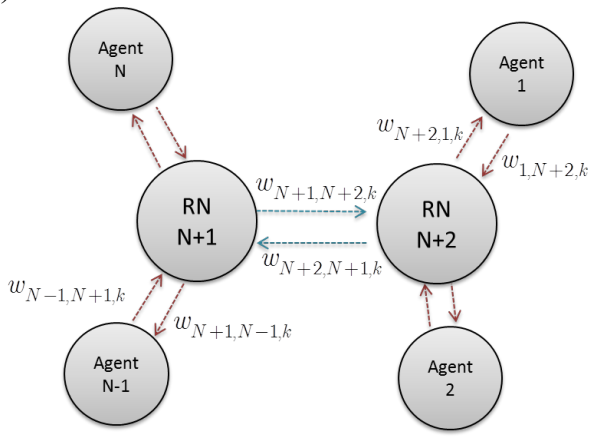

Fig. 7. Star network topology with time varying link weights. Example with two RNs.

The network of agents and relay nodes can be modelled as an undirected graph with a topology sketched in Fig. 7. The whole network has a set of $N+N_{r}$ nodes, $\mathrm{N} \equiv\left\{1,2, \ldots, N+N_{r}\right\}$, with $\left\{N, \ldots, N+N_{r}\right\}$ the indices of 
the relay nodes, and an adjacency matrix given by:

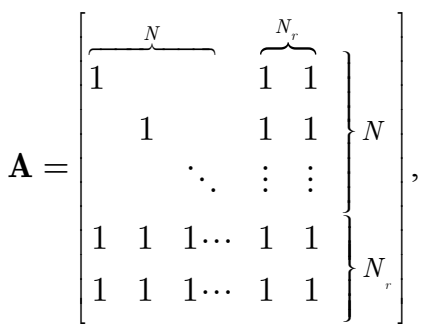

which defines the set of all possible graph edges $\varepsilon \equiv\left\{(i, l) \mid A_{i, l}=1, i, l \in \mathrm{N}\right\}$, with $A_{i, l}$ the $i$-th row and $l$-th column element of A. Actually, the structure of the network is dynamic i.e. at each time step $k$, there is a subset $\varepsilon_{k} \subseteq \varepsilon$ of edges which are active, where an edge $(i, l) \in \varepsilon_{k}$, with $i, l \in \mathrm{N}$, is active if node $i$ can communicate with node $l$. At each $k=m B$, the consensus algorithm is applied to the local estimates of the information matrices, $\hat{\mathbf{D}}_{i, m B}=\hat{\mathbf{C}}_{i, m B}^{-1}$, and the information vectors, $\hat{\mathbf{g}}_{i, m B}=\hat{\mathbf{D}}_{i, m B} \hat{\mathbf{c}}_{i, m B}$, at each node, included the relay node(s):

$$
\begin{aligned}
& \hat{\mathbf{g}}_{i, m B}=\sum_{l \in \mathrm{N}_{i, m B}} w_{i, l, m B} \hat{\mathbf{g}}_{l, m B}, \\
& \hat{\mathbf{D}}_{i, m B}=\sum_{l \in \mathrm{N}_{i, m B}} w_{i, l, m B} \hat{\mathbf{D}}_{l, m B},
\end{aligned}
$$

where $\mathrm{N}_{i, m B}$ is the set of node neighbors of the $i$-th node (the node $i$ is included in the set) at time step $k=m B$ and $w_{i, l, m B}$ are weighting parameters. Once the consensus has been applied, the updated coefficient estimate and the associated covariance for the $i$-th node are $\hat{\mathbf{c}}_{i, m B}=\hat{\mathbf{D}}_{i, m B}^{-1} \hat{\mathbf{g}}_{i, m B}$ and $\hat{\mathbf{C}}_{i, m B}=\hat{\mathbf{D}}_{i, m B}^{-1}$, respectively. The choice of the weights in the consensus update is crucial for guaranteeing certain properties and asymptotic convergence. In particular, in this work, the Metropolis weights are considered:

$w_{i, l, m B}= \begin{cases}1 /\left[1+\max \left(d_{i, m B}, d_{l, m B}\right)\right] & (i, l) \in \varepsilon_{m B} \\ 1-\sum_{l \in \mathrm{N}_{i, m B} \backslash i} w_{i, l, m B} & i=l \\ 0 & \text { otherwise }\end{cases}$

with $d_{i, m B}=\left|\mathrm{N}_{i, m B}\right|$ the cardinality of $\mathrm{N}_{i, m B}$. This choice is average preserving and for certain problems of distributed consensus, it provides asymptotic convergence to a global solution under mild conditions on the sequence of sets of active edges $\varepsilon_{k}$.

In the case of a network of underwater gliders, the $i$-th field sensor applies consensus if it is connected to a relay node, when at surface, after having reached a waypoint. Let define the time step at which the $i$-th sensor reaches the $w$-th waypoint at surface as $k_{i, w}=m_{i, w} B$. The sensor and the connected relay node exchange their current estimates each other (coefficient vector and associated covariance) and locally apply the consensus algorithm. The communication is through a radio or a satellite link. The glider calculates the correction to the navigation heading to point to the next waypoint according to the control law in section II.D and then starts a new cycle of dive/climb phases. The next solution update is applied when the glider reaches the next waypoint at $k_{i, w+1}=m_{i, w} B+\Delta_{i}$ with $\Delta_{i}$ a random variable with given statistical distribution. In this work, $\Delta_{i}$ is a uniform random variable with a given mean value, that is a pre-programmed glider mission parameter usually between 1 and $3 \mathrm{~h}$, within a given interval, typically 15 to $30 \mathrm{~min}$ wide, in order to model the uncertainty due to environmental factors affecting the vehicle navigation (such as unknown water currents).

The consensus update phase in this context is completely asynchronous. With the given network topology, the direct communication between glider agents is not possible. However, sensors indirectly combine their estimates among each other through the relay nodes. The relay nodes allow the diffusion of the information through the network and the convergence of any local agent estimates to the global statistic.

\section{4. RESULTS}

In this section, the system is tested by simulating a network of underwater glider vehicles carrying on board a sensor for measuring environmental parameters, like the seawater temperature. In all tests, the network has one RN. Two simulated scenarios are considered. The first one is static and it includes a sparse field simulated as a sum of Gaussian RBFs with constant coefficients. The second is dynamic and it simulates a field by using the same RBFs as the previous scenario with slowly varying sinusoidal coefficients. Both scenarios are in 3D. Statistics of the network performance are estimated through Mont Carlo simulations. Finally, a realistic non-sparse test case is considered in which the true 3D field is provided by a sea water temperature forecast model. The test case duration is 7 days and it can be considered as a realistic simulation of a glider network operation.

The three scenarios here considered allow testing the system under different conditions related to the characteristics of the spatial field, including dynamic and sparsity of the state vector c. The results are indicative of the general performance of the system in case of i) static and ii) dynamic sparse fields, and iii) dynamic compressible fields.

\section{A. Case study 1: constant coefficients}

In the first test case the true spatial field is modelled in $3 \mathrm{D}$ as the weighted sum of $K=4$ Gaussian RBFs with different mean position vector and the same covariance matrix, $\mathbf{V}=0.05 \mathbf{I}_{3} \quad\left(\mathbf{I}_{3}\right.$ being the $3 \times 3$ identity matrix $)$. The $R B F$ spread parameter is given in normalized coordinates (between 0 and 1). The true non-zero coefficients are constant in time 
and equal to $\mathbf{c}_{\text {true }}=[3,6,9,14]^{T}$.

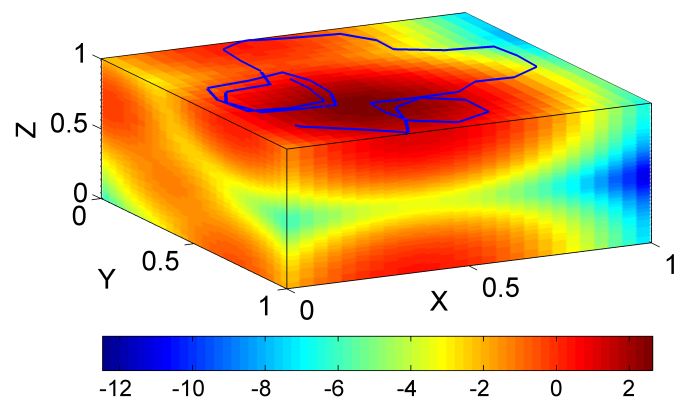

Fig. 8. True sparse field $3 \mathrm{D}$ view simulated as a weighted sum of RBFs. The number of non-zero coefficients is $K=4$ on a total of $L=64$ coefficients associated to 64 Gaussian RBFs distributed on a regular grid. The intensity is in natural logarithmic scale. The blue line represents the trajectory of an agent projected on the horizontal plane at $z=1$.

The field is reconstructed on a $27 \times 27 \times 273 \mathrm{D}$ regular grid representing a spatial domain of $30 \times 30 \mathrm{Km}$ along the $x$ and $y$ directions and $100 \mathrm{~m}$ along the vertical direction. Fig. 8 shows a $3 \mathrm{D}$ view of the true field and an example of the trajectory of an agent projected onto the horizontal plane (the 3D coordinates are normalized between 0 and 1 for convenience).

The sparse modeling of the field is obtained using a dictionary of 64 Gaussian RBFs with mean position vectors distributed on a regular $4 \times 4 \times 43 \mathrm{D}$ sub-grid of the reconstruction grid and with the same known covariance matrix $\mathbf{V}$. The mean positions of the true field RBFs are distributed on four points of this sub-grid (values are not included for brevity). Off-grid effects and different RBFs scale parameters are not considered in this work as they will be the subject for future investigations. The true field coefficient vector, c, is then a 64-dimensional sparse vector (whose entrances correspond to the lexicographical ordering of the RBFs mean position sub-grid), with four components different from zero (the values being equal to the components of $\mathbf{c}_{\text {true }}$ above) and with support depending on the position of the mean of each true field RBF.

According to (2), the state equation for a single agent Kalman filter is:

$\mathbf{c}_{k}=\mathbf{I}_{64} \mathbf{c}_{k-1}+\mathbf{n}_{k}$,

with the process noise covariance matrix set to $\mathbf{Q}_{k}=0.003 \mathbf{I}_{64}$ and where $I_{64}$ is the $64 \times 64$ identity matrix. No exogenous inputs are considered. In this first test and in the next ones (see sections IV.B and IV.C), the true state model is considered unknown to the network. The process noise in (31) represents the uncertainty due to a not well defined state equation the introduction of which allows tracking slowly-time variations of the state vector in case of a model mismatch. The measurement equation of each agent is (11) with measurement noise equal to $R_{k}=0.001$. The task of the distributed system is to estimate the state vector $\mathbf{c}$ (i.e. coefficient amplitudes and vector support) given the noisy measurements of the true field.

The network includes $N=15$ glider agents with the same kinematic characteristics (the kinematic is constrained as in the glider case in Section III) and measurement sensors. The agent speed is $0.6 \mathrm{~m} / \mathrm{s}$, the pitch angle is $\phi=26^{\circ}$ the sampling rate is $T=6 \mathrm{~s}$ and the CS block length is $B=5$ (that is one CS measurement every $30 \mathrm{sec}$ and about $11 \mathrm{CS}$ measurements along the water column in a single climb or dive phase). The chip sequence of the CS device is chosen at random according to subsection II.B. The SA-KF parameters are $\lambda_{S A}=10^{-3}, \zeta_{S A}=10^{-3}$, and $\gamma_{S A}=10^{-5}$, while the number of iterations is 16 . The time delay between adjacent surfacing/transmission phases of an agent is modelled as a uniform random variable $\Delta \in\left[\eta-\delta_{s}, \eta+\delta_{s}\right]$, to take into account random fluctuations due to unknown environmental conditions (sea current) affecting the agent navigation. The average delay $\eta$ is typically between 1-3 hours while in all the simulations $\delta_{s}$ is set to $15 \mathrm{~min}$. The duration is $T_{D}=72 \mathrm{~h}$.

Initially, the agents are placed uniformly at random in the considered domain and the initial value of the coefficient estimates is supposed to be a Gaussian random variable with zero mean and 0.5 standard deviation. Performance statistics have been evaluated by carrying out 100 Monte Carlo runs. The performances are evaluated in terms of the steady state field root mean square error (RMSE) as a function of the ratio $\gamma=T_{D} / \eta \quad$ (the total number of connections in the observation period). The RMSE curve is compared against the ideal case of $\gamma \rightarrow+\infty$ and $\delta_{s} \rightarrow 0$, i.e. the centralized solution as in Section II-A. The network has been simulated for different values of the mean transmission delay parameter $\eta \in\{0.8 \mathrm{~h}, 1.2 \mathrm{~h}, 1.6 \mathrm{~h}, 2 \mathrm{~h}, 2.4 \mathrm{~h}, 3 \mathrm{~h}\}$. The values of $\eta$ are compatible with the kinematic and the track geometry of glider vehicles allowing at least one dive-climb cycle.

Fig. 9 shows the comparison in terms of steady state field RMSE as function of $\gamma=T_{D} / \eta$ among different sparsity aware Kalman filters as described in section II.C. In particular, the SAKF1 is the algorithm proposed in this work as a slight variant of the original SA-KF proposed in [15][16], here referred to as SAKF2. A third implementation, here called SAKF3, is based on pseudo measurements norms as proposed in [31]. The versions using both $L_{1}$ and $\mathrm{SL}_{0}$ norms are also compared. The algorithms run using the same number of iterations and the performance is averaged over 100 Monte Carlo simulations. The RMSE scale of SAKF1 and SAKF2 is reported on the left axis while the scale of the SAKF3 on the right axis. The SAKF1 outperforms SAKF2 and SAKF3, with a slight improvement of the $\mathrm{SL}_{0}$-norm version with respect to the $L_{1}$-norm one, for values of $\gamma$ which are typical of a real glider mission setup (e.g. $\gamma=45$ ). The SAKF1 and SAKF2 outperform the SAKF3 by two orders of magnitude. The SAKF1 RMSE is almost constant in the considered $\gamma$ interval with a standard deviation that is about 6 times lower than the SAKF2, showing better solution stability $( \pm 2 \sigma$ confidence levels are not reported in the graph for clarity). The SAKF1 has been chosen to run the simulations reported in the sequel of this section and in sections IV.B and IV.C. 


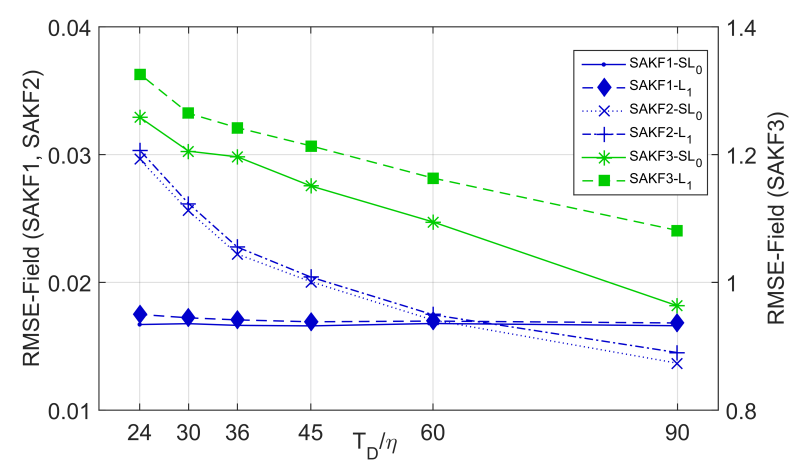

Fig. 9. Steady state field RMSE. SA-KF algorithm comparison using 16 iterations. Performance averaged over 100 Monte Carlo simulations.

Fig. 10 shows the steady state field RMSE at the RN averaged over 100 Monte Carlo runs as function of $\gamma=T_{D} / \eta$ with $2 \sigma$ bars (blue line) compared versus the RMSE of the centralized solution (in red). As expected, in the static case, the steady state error does not depend significantly on $\gamma$ within the range of values considered in the simulation. Its mean value is around 0.0167 . The achieved average relative error is on the order of $2 \%$.

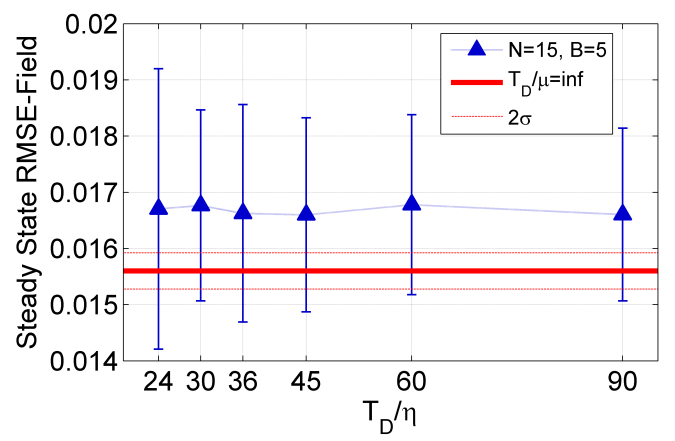

Fig. 10. Steady state field RMSE at the RN as function of $\gamma=T_{D} / \eta$ compared with the centralized consensus solution. Constant coefficient case.

Fig. 11 depicts the estimation of the field at the end of the observation period showing the good match with true field in Fig. 8.

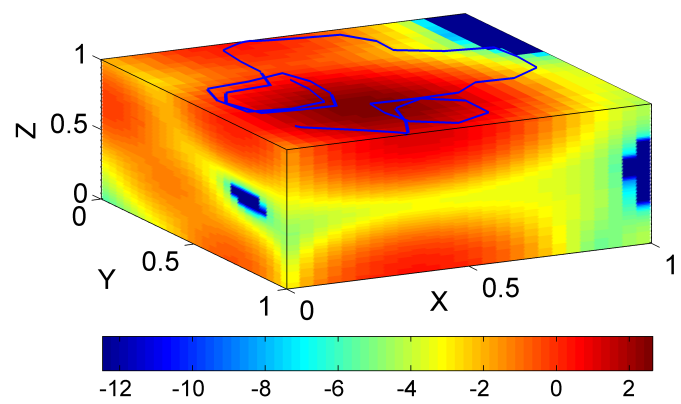

Fig. 11. Estimated field (natural logarithmic scale) at the end of the observation period with optimal trajectory for sensor 15 projected on the horizontal plane.

Fig. 12 provides the field RMSE versus time for $\gamma=45$ ( $\eta=1.6 \mathrm{~h}$ ), averaged over 100 Monte Carlo runs, for both the $\mathrm{RN}$ and the sensors. After a transitory phase of about $48 \mathrm{~h}$, the sensors (in blue) achieve a consensus and the RMSE converge to the same value on average. The RMSE of the RN follows the same dynamic with $2 \sigma$ confidence levels converging as well to a steady state value.

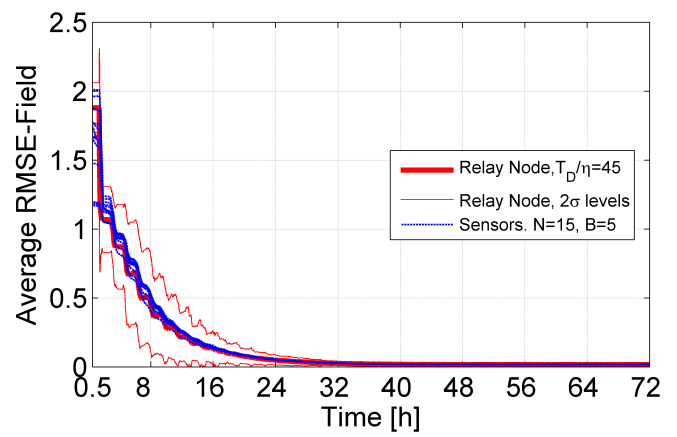

Fig. 12. Field RMSE (averaged over 100 Monte Carlo runs) versus time of the $\mathrm{RN}$ and the sensors for $\gamma=45$.

Fig. 13 shows the steady state phase of the graph in Fig. 12 confirming the convergence of the sensors and the RN RMSE. The RN average error slightly improves with respect to the mean of RMSE of sensors. The $2 \sigma$ confidence intervals are close to each other and therefore only the $\mathrm{RN}$ is reported.

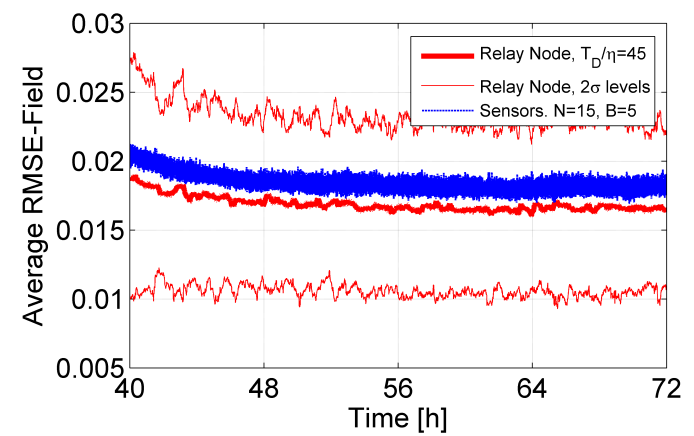

Fig. 13. Field RMSE (averaged over 100 Monte Carlo runs) versus time of the $\mathrm{RN}$ and the sensors for $\gamma=45$. Zoom in of the steady state phase.

Fig. 14 finally depicts the estimate of a non-zero coefficient versus time for the sensors and the $\mathrm{RN}(\gamma=45)$. The sensors estimates are initially very different producing oscillations in the $\mathrm{RN}$ estimate. The sensor and the $\mathrm{RN}$ estimates gradually converge to the true coefficient achieving consensus on average after about $48 \mathrm{~h}$.

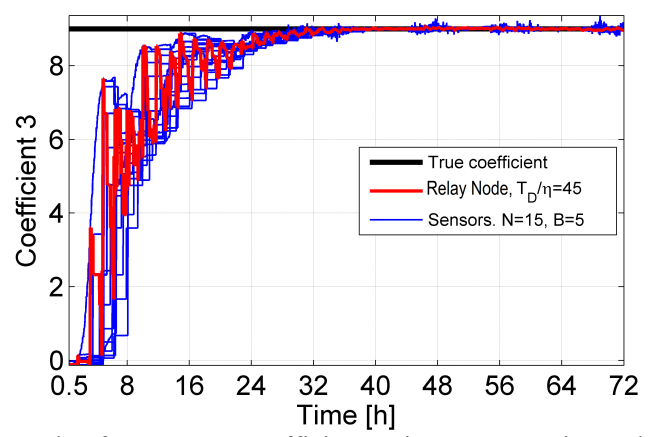

Fig. 14. Example of a non-zero coefficient estimate versus time. The sensors and the RN gradually reach the consensus after about 48 hours.

\section{B. Case study 2: sinusoidal coefficients}

In the second scenario, the field is modeled as in the previous case except for the coefficient amplitudes that are 
sinusoidal with the mean value as in the constant case and the amplitude that is $5 \%$ of the mean. The periods of the four sinusoidal components are set to $T_{P} \in\{6 \mathrm{~h}, 7 \mathrm{~h}, 8 \mathrm{~h}, 9 \mathrm{~h}\}$. The parameters of the simulation are the same of the constant case including the Gaussian RBF dictionary, agent model, Kalman state and measurement equations for each agent, number of agents and statistical properties of the transmission delay. The average performance is evaluated over 100 Monte Carlo runs versus $\gamma$ with the same time horizon $T_{D}$ and $\eta$ of the previous case.

Fig. 15 shows the steady state RMSE of the field for the RN (in blue) compared with the full centralized solution (in red). Differently from the previous case, in a dynamic scenario, the RMSE is much more affected by the value of $\eta$.

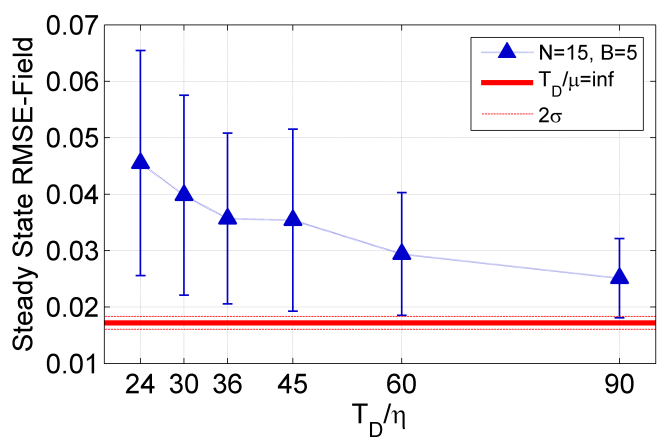

Fig. 15. Steady state field RMSE at the RN as function of $\gamma=T_{D} / \eta$ compared with the centralized consensus solution. Sinusoidal coefficient case.

In particular, the error at $\gamma=24(\eta=3 \mathrm{~h})$ is roughly twice the error at $\gamma=90 \quad(\eta=0.8 \mathrm{~h})$, asymptotically decreasing as $\gamma$ varies between these two limits toward the ideal fully centralized solution. The average relative error is in general of the same order of magnitude of the constant case.

Fig. 16 shows the field estimation of the RN (in red) and the sensors (in blue) versus time compared with the real field at a given position $(x=0.54, y=0.31$ and $z=0.46$, in normalized coordinates). The network and the $\mathrm{RN}$ achieve a consensus, in average, after a transitory of about 24 hours in which the local estimates are very different from each other. After the transitory, the network try to tracks the true field variations with an error that depends on $\gamma$ (in the example of Fig. 16 $\gamma=60, \eta=1.2 \mathrm{~h})$.

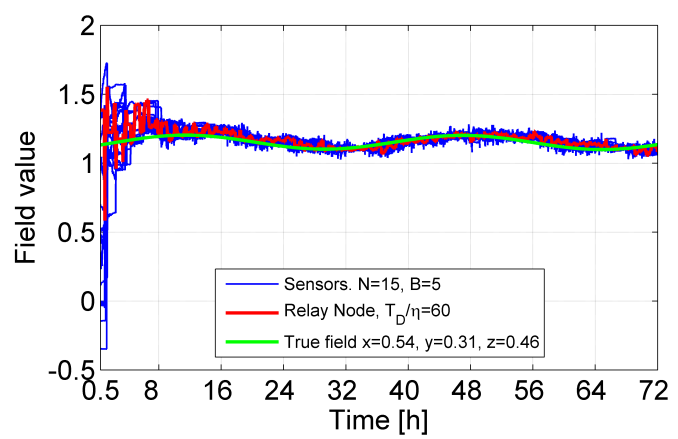

Fig. 16. Estimated field from sensors and fusion center at given position.

Fig. 17 depicts the field RMSE versus time for the same $\gamma=60(\eta=1.2 \mathrm{~h})$, averaged over 100 Monte Carlo runs, for both the RN and the sensors. The sensors (in blue), after the transitory phase of $24 \mathrm{~h}$, achieve a consensus with the RMSE converging to the same value on average. The RMSE of the $\mathrm{RN}$ (in red) follows the same dynamic with $2 \sigma$ confidence levels converging as well to a steady state.

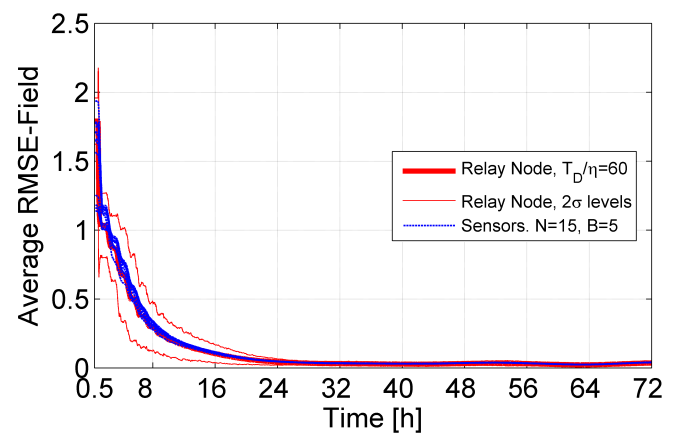

Fig. 17. Decentralized, sinusoidal coefficients, glider case. Temporal Field RMSE.

From Fig. 18, we get that, differently from the static case, the error in the steady phase presents a residual bounded oscillation around a constant value. As confirmed by Fig. 15 the mean and the amplitude of the residual oscillation decreases as $\gamma$ increases (see both the mean value and the $2 \sigma$ error bars) i.e. the network is improving the capability of tracking the real underline field dynamic.

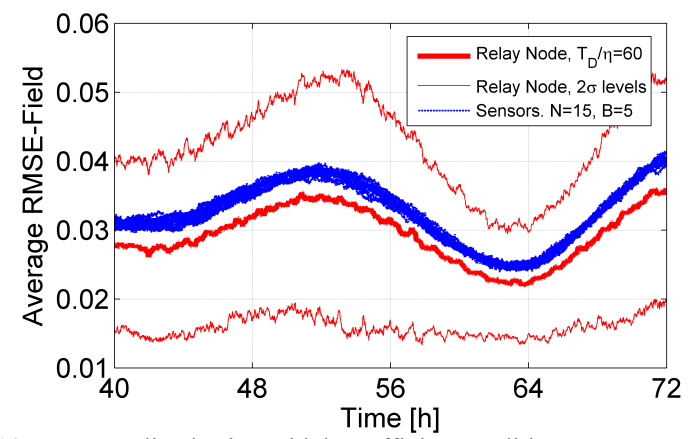

Fig. 18. Decentralized, sinusoidal coefficients, glider case. Temporal Field RMSE. Steady state phase.

Finally, Fig. 19 shows the estimate of a non-zero coefficient versus time for the sensors and the RN (for the same case of $\gamma=60)$. As in the constant case, the sensors and the RN estimates, initially with different dynamics, gradually achieve a consensus (in roughly 24 hours) and in the steady phase they track the true coefficient.

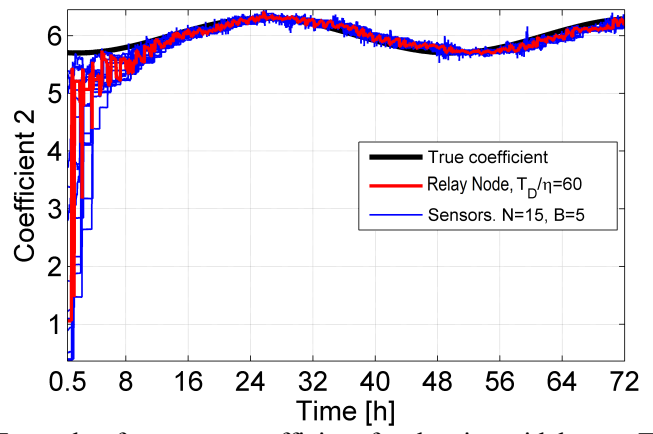

Fig. 19. Example of non-zero coefficient for the sinusoidal case. The sensors and the fusion center gradually reach a consensus after 16 hours and track the coefficient variations. 


\section{NCOM water temperature forecasts.}

In a third scenario, the network task is the estimation of a non-sparse dynamic field. The true field is constructed by sequencing a series of consecutive 3D forecasts of sea water temperature (with $3 \mathrm{~h}$ sampling period) of the Navy Coastal Ocean Model [18], spanning an observation period of 7 days. The model was provided by the Naval Research LaboratoryStannis Space Centre (NRL-SSC), during the STO-CMRE 2011 Recognized Environmental Picture cruise trial (REP11) in the Mediterranean Sea. The data set used in the simulation represents a sub-volume of about 60 by $60 \mathrm{Km}$ in the horizontal plane by $100 \mathrm{~m}$ along depth. The horizontal resolution is about 2 by $2 \mathrm{Km}$. The initial depth levels (not regularly spaced) have been linearly interpolated between 0 and $100 \mathrm{~m}$. The resulting regular data grid has a size of $30 \times 30 \times 30$ samples.

Fig. 20 shows an example of the true NCOM field at the end of the observation period. Without loss of generalities, the spatial/temporal mean of the field has been subtracted from the original data set. The system reconstructs the variations around that constant value.

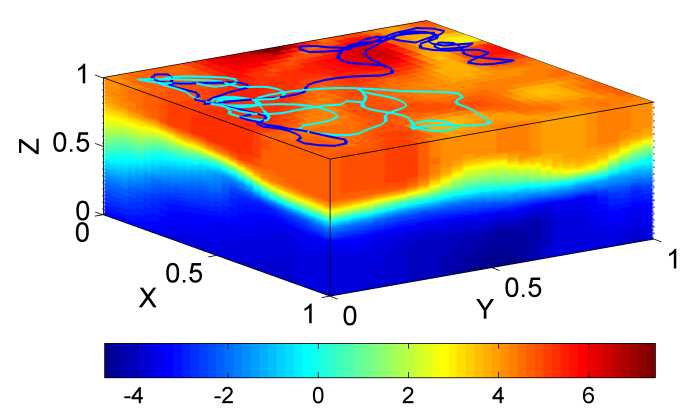

Fig. 20. Example of NCOM sea water temperature field variations at the end of the 7 days observation period and optimal trajectories projected on the horizontal plane of two agents. Temperature variations are in ${ }^{\circ} \mathrm{C}$ around the global mean.

The simulation set up is the same as in the previous cases except for the basis function dictionary that is of 343 Gaussian RBFs (i.e. the state coefficient vector c has 343 entries) arranged on a $7 \times 7 \times 73 \mathrm{D}$ regular sub-grid of the original $30 \times 30 \times 30$ NCOM model grid. The RBF covariance matrix is $\mathbf{V}=0.025 \mathbf{I}_{3}$ constant for all the dictionary functions. The RBF spread parameter was chosen empirically by roughly estimating the spatial scale of the main oceanographic features present in the data. The results are provided for a single realization as the scenario does not allow a series of Monte Carlo simulations in a reasonable time as in the previous simulated cases.

Fig. 21 presents the $3 \mathrm{D}$ view of the reconstruction of the field at the end of the observation period showing a good match with the true field in terms of the main oceanographic features both in the horizontal plane and along the vertical water column. The results are for a fleet of $N=15$ gliders with $B=5$ and $\eta=1$ h (i.e. $\gamma=168$ ).

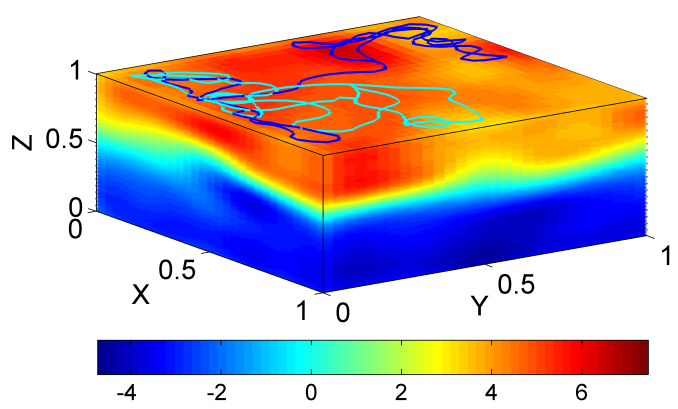

Fig. 21. Sea water temperature reconstructed field at the end of the observation period for the NCOM case. Temperature variations are in ${ }^{\circ} \mathrm{C}$ around the global mean.

Fig. 22-a shows the sensors and the RN field estimates versus time (sub-sampled every 3 hours) for a given spatial position (with $x=0.79, y=0.66, z=1.00$ ) compared with the true field (in green). In this case, the system starts to track the field variations after a transition phase of about 18 hours. In the case depicted in Fig. 22-b (field versus time at position $x=0.69, y=0.07, z=1.00$ ), the transition phase is longer, roughly 72 hours, after that the sensors and the RN start to track the field variations more closely.

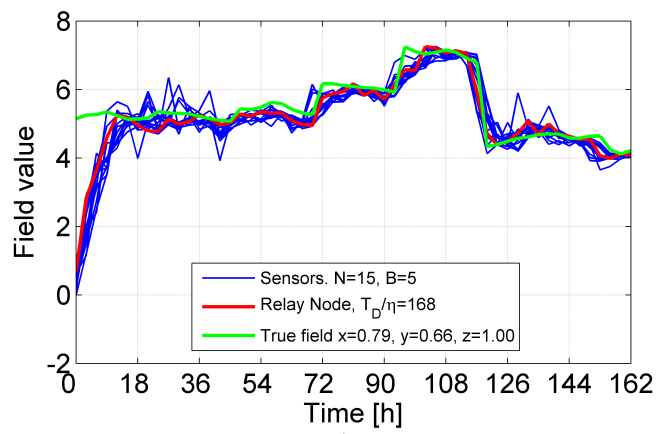

a)

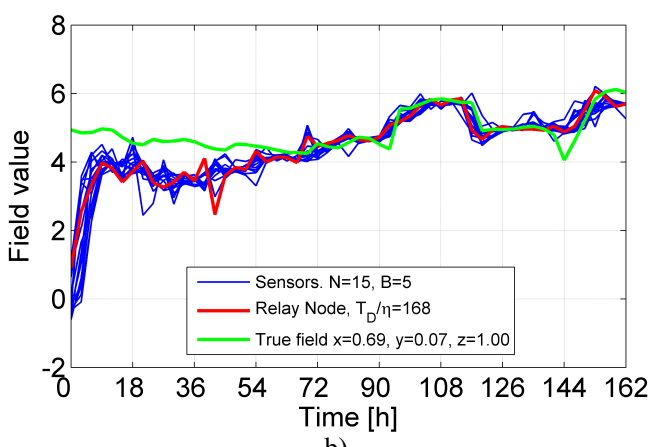

b)

Fig. 22. Sensors and RN estimated field at a given position sub-sampled every 3 hours. a) The transition phase duration of the field estimates is about $18 \mathrm{~h}$ after that the estimates track the true NCOM field variations. b) The transition phase duration in this second case is about $72 \mathrm{~h}$ after that the estimates start to track the true NCOM field variations more closely.

Fig. 23-a shows the vertical profiles of the true NCOM field along the trajectory of the agent 1 while Fig. 23-b depicts the estimated profile by the same agent. The main oceanographic features are well resolved as well as the thermocline separating water masses with different characteristics. 


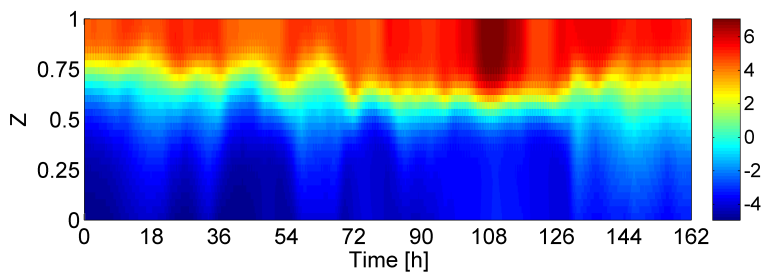

a)

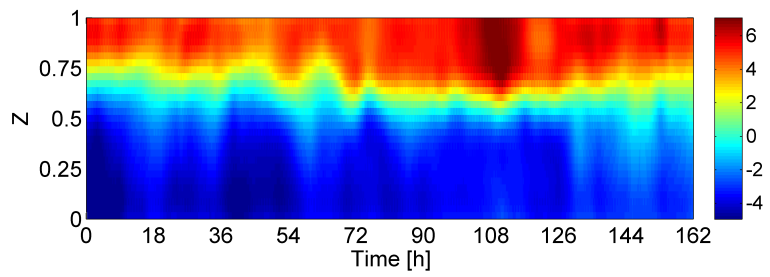

b)

Fig. 23. a) NCOM water temperature field vertical profile along agent 1 track (normalized $z$ coordinates). b) Estimated temperature field vertical profile along agent 1 track.

Fig. 24 shows the spatial field RMSE versus time for different values of the $\mathrm{N}$ and $\eta$ parameters. The graph can provide some indications on the best parameters to use in a real scenario, having the same time and spatial scale, and variability, in order to achieve the best possible performance at an affordable cost (in terms of number of sensors and number of transmissions in the observation period). In particular, the number of sensors significantly affects the error and the adaptation capability of the system for $N<15$. After that limit, the RMSE value and the adaptation are almost stable (close to $0.5{ }^{\circ} \mathrm{C}$ ), and the $\eta$ parameter does not significantly affect the performance. The spatial percentage error of the field is close to $10 \%$ after the transitory phase and for $N \geq 15$.

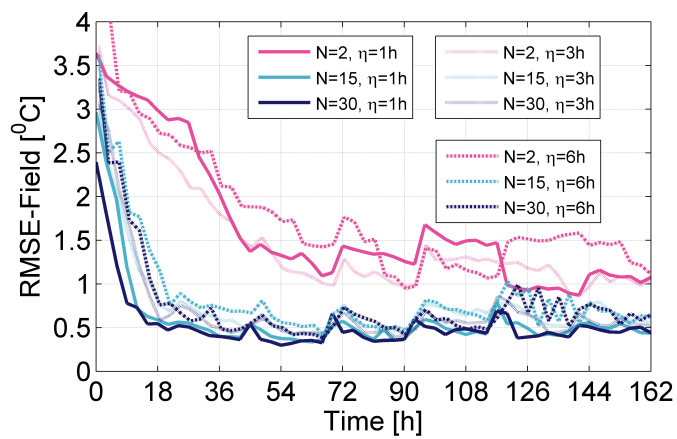

Fig. 24. Spatial field RMSE versus time for different values of $N$ and $\eta$ (3h subsampling).

\section{CONCLUSIONS}

In this paper, an autonomous dynamic sensor network is proposed and tested on a scenario simulating the adaptive control of a fleet of autonomous underwater gliders. The task of the sensor network is to estimate $3 \mathrm{D}$ spatial scalar fields of ocean variables. The communication constrains and the sporadic asynchronous communications are the main operational limiting factors of these kinds of underwater networks. In order to cope with these issues, the proposed architecture manages in a distributed fashion both the field estimation and the agent control. Each agent performs the local estimation of the field statistics by a Kalman filter that processes local field measurements. The local estimates of an agent are updated, through an asynchronous consensus algorithm, exploiting the local estimates of the others. The information exchange is possible due to a sub-network of relay nodes that communicate with the agents at surface. The relay nodes apply consensus (asynchronously) to the local estimates of the agents and among them so as to provide an updated global field estimate that is exchanged with the agents themselves. In this way, all the nodes of the network converge on average to the global field statistics. This allows an agent to use global information to control its position in order to acquire field measurements in the most informative areas and adaptively track the field variations. The processing scheme can also take into account the spatial sparsity of the field by including at the agent level a SA-KF that refines the solution imposing $L_{0}$-norm sparsity constraints. Moreover, the local sampling scheme is based on a CS sampling device (such as the random demodulator) to reduce the sampling rate and retain the field information. The CS samples are directly used in the local Kalman filter, on-line, without the need of reconstructing the measurement sequence at the original sampling rate.

The system has been tested on simulated scenarios both sparse and non-sparse. In the tests, the field is modeled by a dictionary of Gaussian RBFs. The first two scenarios include a constant and a dynamic sparse field while a third scenario simulates a glider fleet mission of 7 days using 3D timevarying sea water temperature data provided by the NCOM forecast model for a Mediterranean Sea area, in the framework of the REP11 experiment. For typical fleet operational parameters (i.e. 15 agents and 1-3 hours surfacing period) and surveyed area size $(30 \times 30$ or $60 \times 60 \mathrm{Km}$ horizontally and 100 $m$ vertically) the average performance achieved in terms of relative error at steady state is within $10 \%$.

Future work can be directed in several ways. The on-line estimation of base functions unknown parameters, such as the mean and the covariance of Gaussian RBFs, is of particular importance in real applications. The problem is challenging as involves non-linerities that complicate the estimation step and also the agent control law. Further work includes the investigation of the effects of the water current affecting the agent navigation.

\section{ACKNOWLEDGMENT}

NCOM forecasts are provided by the US Naval Research Laboratory - Stennis Space Center (NRL-SSC) in the framework of the STO-CMRE REP11 experiment.

\section{REFERENCES}

[1] Schofield, O., J. Kohut, D. Aragon, L. Creed, J. Graver, C. Haldeman, J. Kerfoot, H. Roarty, C. Jones, D. Webb, and S.M. Glenn., "Slocum gliders: Robust and ready," Journal of Field Robotics 24(6):1-14, 2007.

[2] Alvarez, A.; Garau, B.; Caiti, A., "Combining networks of drifting profiling floats and gliders for adaptive sampling of the Ocean," 2007 IEEE International Conference on 2007 IEEE International Conference on Robotics and Automation, vol., no., pp.157-162, 10-14 April 2007.

[3] Fiorelli, E.; Leonard, N.E.; Bhatta, P.; Paley, D.A.; Bachmayer, R.; Fratantoni, D.M., "Multi-AUV Control and Adaptive 
Sampling in Monterey Bay," Oceanic Engineering, IEEE Journal of , vol.31, no.4, pp.935,948, Oct. 2006.

[4] Leonard, N.E.; Paley, D.A.; Lekien, F.; Sepulchre, R.; Fratantoni, D.M.; Davis, R.E., "Collective Motion, Sensor Networks, and Ocean Sampling," Proceedings of the IEEE, vol.95, no.1, pp.48,74, Jan. 2007.

[5] Sheng-Yuan Tu; Sayed, A.H., "Mobile Adaptive Networks," Selected Topics in Signal Processing, IEEE Journal of , vol.5, no.4, pp.649,664, Aug. 2011.

[6] Sayed, A.H.; Sheng-Yuan Tu; Jianshu Chen; Xiaochuan Zhao; Towfic, Z.J., "Diffusion strategies for adaptation and learning over networks: an examination of distributed strategies and network behavior," IEEE Signal Processing Magazine, vol.30, no.3, pp.155,171, May 2013.

[7] Olfati-Saber, R.; Fax, J.A.; Murray, R.M., "Consensus and Cooperation in Networked Multi-Agent Systems," Proceedings of the IEEE, vol.95, no.1, pp.215-233, Jan. 2007.

[8] Olfati-Saber, R., "Flocking for multi-agent dynamic systems: algorithms and theory," IEEE Transactions on Automatic Control, vol.51, no.3, pp.401-420, March 2006.

[9] P. Braca, R. Goldhahn, K. LePage, S. Marano, V. Matta and P. Willett, "Cognitive Multistatic AUV Networks," in Proc. of the 17th International Conference on Information Fusion (FUSION 2014), Salamanca 2014.

[10] P. Braca, S. Marano, V. Matta and P. Willett, "Asymptotic Optimality of Running Consensus in Testing Binary Hypotheses," IEEE Transactions on Signal Processing, vol.58, no.2, pp.814,825, Feb. 2010.

[11] P. Braca, S. Marano, and V. Matta, "Enforcing Consensus While Monitoring the Environment in Wireless Sensor Networks," IEEE Transactions on Signal Processing, vol. 56, no. 7 pp.3375,3380, July 2008.

[12] Kar, S.; Moura, J.M.F., "Consensus + innovations distributed inference over networks: cooperation and sensing in networked systems," IEEE Signal Processing Magazine, vol.30, no.3, pp.99-109, May 2013.

[13] Hine, R.; Willcox, S.; Hine, G.; Richardson, T., "The Wave Glider: A Wave-Powered autonomous marine vehicle," OCEANS 2009, MTS/IEEE Biloxi - Marine Technology for Our Future: Global and Local Challenges, vol., no., pp.1,6, 26-29 Oct. 2009.

[14] Grasso, R., Braca, P., Fortunati, S., Gini, F., Greco, M., S., "Distributed Underwater Glider Network with Consensus Kalman Filter for Environmental Field Estimation," Proceedings OCEANS 2015 MTS/IEEE, Genova, Italy, 2015.

[15] Farahmand, S., Giannakis, G., B., Leus, G., Tian, Z., "Sparsityaware Kalman Tracking of Target Signal Strengths on a Grid," Information Fusion (FUSION), 2011 Proceedings of the 14th International Conference on, pp.: 1 - 6, 2011.

[16] Farahmand, S., Giannakis, G., B., Leus, G., Tian, Z., "Tracking target signal strengths on a grid using sparsity," EURASIP Journal on Advances in Signal Processing, Springer International Publishing, Jan 2014, doi: 10.1186/1687-61802014-7.

[17] Tropp, J.A.; Laska, J.N.; Duarte, M.F.; Romberg, J.K.; Baraniuk, R.G., "Beyond Nyquist: Efficient Sampling of Sparse Bandlimited Signals," IEEE Transactions on Information Theory, vol.56, no.1, pp.520-544, Jan. 2010.

[18] Martin, P. J., "A description of the Navy Coastal Ocean Model Version 1.0," NRL Rep. NRL/FR/7322-00-9962, 42 pp., NRL, Stennis Space Center, MS, USA, 2000.

[19] K. M. Lynch et al, "Decentralized Environmental Modeling by Mobile Sensor Networks," IEEE Transactions on Robotics, Vol. 24, No 3, June 2008.

[20] Hung Manh La; Weihua Sheng, "Distributed Sensor Fusion for Scalar Field Mapping Using Mobile Sensor Networks," IEEE
Transactions on Cybernetics, vol.43, no.2, pp.766, 778, April 2013.

[21] Chang, K., C.; Saha, R., K.; Bar-Shalom, Y., "On Optimal Track to-Track Fusion," IEEE Transactions on Aerospace and Electronic Systems, vol. 33, no. 4, Oct. 1997.

[22] Tian, X.; Bar-Shalom, Y., "Track-to-Track Fusion Configurations and Association in a Sliding Window," Journal of Advances in Information Fusion, vol. 4, no. 2, Dec 2009.

[23] Uhlmann, J., K., "Covariance consistency methods for faulttolerant distributed data fusion," Information Fusion, no. 4, pp 201-215, 2003.

[24] Elad, M., Yavneh, I., "A Plurality of Sparse Representations Is Better Than the Sparsest One Alone," IEEE Transactions on Information Theory, vol. 55, no. 10, Oct. 2009.

[25] Brito, M., P., Smeed, D., A., Griffiths, G., "Analysis of causation of loss of communication with marine autonomous systems: A probability tree approach," Methods in Oceanography, vol. 10, September 2014, Pages 122-137, ISSN 2211-1220, http://dx.doi.org/10.1016/j.mio.2014.07.003.

[26] Julier, S., J., Uhlmann, J., K., "Unscented Filtering and Nonlinear Estimation," Proceedings of the IEEE, Vol. 92, No. 3, March 2004.

[27] Merwe, R. van der, "Sigma-Point Kalman Filters for Probabilistic Inference in Dynamic State-Space Models," PhD dissertation, Oregon Health \& Science University, April 2004.

[28] Donoho, D.L.; "Compressed sensing," IEEE Transactions on Information Theory, vol. 52, no. 4, pp. 1289-1306, April 2006.

[29] Candes, E.J.; Romberg, J.; Tao, T.; "Stable signal recovery from incomplete and inaccurate measurements", Communications on Pure and Applied Mathematics, vol. 59, no. 8, pp. 1207-1223, August 2006.

[30] J. Mohimani, H.; Babaie-Zadeh, M.; Jutten, C.; "A Fast Approach for Overcomplete Sparse Decomposition Based on Smoothed Norm," IEEE Trans. Signal Process., vol.57, no.1, pp.289-301, Jan. 2009.

[31] A. Carmi, P. Gurfil, and D. Kanevsky, "Methods for sparse signal recovery using Kalman filtering pseudo-measuremennt norms and quasi-norms," IEEE Transactions on Signal Processing, vol. 58, no. 4, pp. 2405-2409, Apr 2010.

[32] Grasso R, Cecchi D, Cococcioni M, Trees C, Rixen M, Alvarez A, Strode C, "Model based decision support for underwater glider operation monitoring," Proceedings OCEANS 2010 MTS/IEEE, Seattle. pp. 1-8, 2010.

[33] Olfati-Saber, R.; Murray, R.M., "Consensus problems in networks of agents with switching topology and time-delays," IEEE Transactions on Automatic Control, vol.49, no.9, pp.15201533, Sept. 2004.

[34] Olfati-Saber, R., "Distributed Kalman filtering for sensor networks," 2007 46th IEEE Conference on Decision and Control, vol., no., pp.5492-5498, 12-14 Dec. 2007.

[35] Olfati-Saber, R., "Distributed Kalman Filter with Embedded Consensus Filters," 44th IEEE Conference on , Decision and Control, 2005 and 2005 European Control Conference. CDCECC '05. vol., no., pp.8179-8184, 12-15 Dec. 2005, doi: 10.1109/CDC.2005.1583486. 


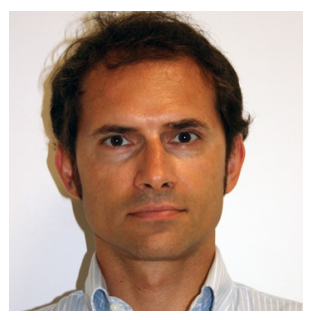

Raffaele Grasso received the Laurea degree in telecommunication engineering from the University of Pisa, Pisa, Italy, in 1996, the Ph.D. in remote sensing from the University of Florence, Florence, Italy, in 2000 and the Executive MBA from the LUISS Business School, Rome, Italy, in 2010. He is scientist in the Research Department at the NATO Science \& Technology Organization-Centre for Maritime Research and Experimentation (CMRE). His current research interests include statistical signal processing, compressive sensing, decision support systems and optimal sensor network deployment and control, with applications in surveillance, environmental monitoring and maritime operation planning. He is coauthor of more than 70 publications in international scientific journals and conference proceedings.

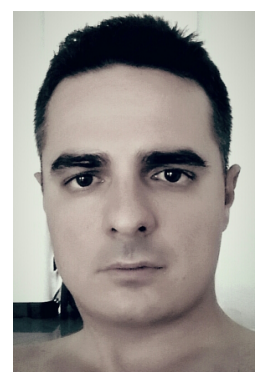

Paolo Braca (M'14) received the Laurea degree (summa cum laude) in electronic engineering, and the Ph.D. degree (highest rank) in information engineering from the University of Salerno, Salerno, Italy, in 2006 and 2010, respectively. In 2009, he was a Visiting Scholar with the Department of Electrical and Computer Engineering, University of Connecticut, Storrs, CT, USA. In 2010, he was a Senior Engineer with D'Appolonia S.p.A., Rome, Italy. In 2010-2011, he was a Postdoctoral Associate with the University of Salerno, Salerno, Italy. In 2011, he joined the NATO Science \& Technology Organization Centre for Maritime Research and Experimentation (CMRE) as a Scientist with the Research Department. His research interests include statistical signal processing with emphasis on distributed inference, sensor networks, adaptation and learning over networks, multitarget/multisensor tracking and data fusion. He is coauthor of more than 70 publications in international scientific journals and conference proceedings. Dr. Braca is currently an Associate Editor of the IEEE Signal Processing Magazine (E-Newsletter) and the Journal of Advances in Information Fusion, and Reviewer for several scientific journals and conferences. He is in the Technical Committee of the major international conferences in the field of signal processing and data fusion. He has been a CoOrganizer with Prof. P. K. Willett of the special session Multisensor Multitarget Tracking at EUSIPCO 2013. He was the recipient of the Best Student Paper Award (first runner-up) at the 12th Conference on Information Fusion in 2009.

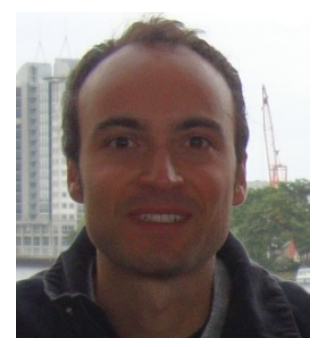

Stefano FORTUNATI graduated in telecommunication engineering and received the $\mathrm{PhD}$ at the University of Pisa, Italy, in 2008 and 2012 respectively. In 2012, he joined the Department of Ingegneria dell'Informazione of the University of Pisa, where he is currently working as temporary researcher. His general interests are in the areas of statistical signal processing, estimation, and detection theory. In particular, his research interests include robust estimation and detection theory, performance bounds, data fusion, target detection in nonGaussian clutter and CFAR techniques.

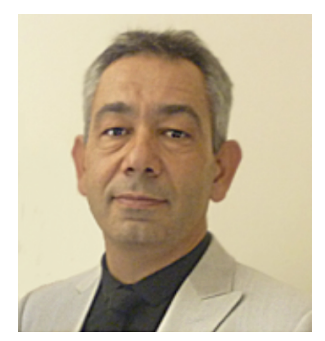

Fulvio Gini (Principal Investigator, Fellow IEEE) received the Doctor Engineer (cum laude) and the Research Doctor degrees in electronic engineering from the University of Pisa, Italy, in 1990 and 1995 respectively. In 1993 he joined the Department of Ingegneria dell'Informazione of the University of Pisa, where he become Associate Professor in 2000 and he is Full Professor since 2006. From July 1996 through January 1997, he was a visiting researcher at the Department of Electrical Engineering, University of Virginia, Charlottesville. $\mathrm{He}$ is an Associate Editor for the IEEE Transactions on Aerospace and Electronic Systems and for the Elsevier Signal Processing journal. He has been AE for the Transactions on Signal Processing (2000-06) and a Member of the EURASIP JASP Editorial Board. He has been the Editor-in-Chief of the Hindawi International Journal on Navigation and Observation (IJNO). He is the Area Editor for the Special issues of the IEEE Signal Processing Magazine. He was co-recipient of the 2001 IEEE AES Society's Barry Carlton Award for Best Paper. He was recipient of the 2003 IEE Achievement Award for outstanding contribution in signal processing and of the 2003 IEEE AES Society Nathanson Award to the Young Engineer of the Year. He has been a Member of the Signal Processing Theory and Methods (SPTM) Technical Committee (TC) of the IEEE Signal Processing Society and of the Sensor Array and Multichannel (SAM) TC for many years. $\mathrm{He}$ is a Member of the Board of Directors (BoD) of the EURASIP Society, the Award Chair (2006-2012) and the EURASIP President for the years 2013-2016. He was the Technical co-Chair of the 2006 EURASIP Signal and Image Processing Conference (EUSIPCO), Florence, Italy, September 2006, of the 2008 Radar Conference, Rome, Italy, May 2008, and of the IEEE CAMSAP 2015 workshop, to be held in Cancun, Mexico in December 2015. He was the General co-Chair of the 2nd Workshop on Cognitive Information Processing (CIP2010), of the IEEE ICASSP 2014, held in Florence in May 2014, and of the CoSeRa 2015 workshop on compressive sensing in radar, held in Pisa in June 2015. He was the guest co-editor of the special section of the Journal of the IEEE SP Society on Special Topics in Signal Processing on "Adaptive Waveform Design for Agile Sensing and Communication" (2007), guest editor of the special section of the IEEE Signal Processing Magazine on "Knowledge Based Systems for Adaptive Radar Detection, Tracking and Classification" (2006), guest co-editor of the two special issues of the EURASIP Signal Processing journal on "New trends and findings in antenna array processing for radar" (2004) and on "Advances in Sensor Array Processing (in memory of Alex Gershman)" (2013). He is co-editor and author of the book "Knowledge Based Radar Detection, Tracking and Classification" (2008) and of the book "Waveform Diversity and Design" (2012). His research 
interests include modeling and statistical analysis of radar clutter data, non-Gaussian signal detection and estimation, parameter estimation and data extraction from multichannel interferometric SAR data. He authored or co-authored 8 book chapters, about 120 journal papers and more than 150 conference papers.

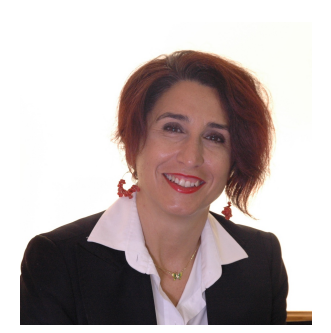

Maria S. Greco graduated in Electronic Engineering in 1993 and received the Ph.D. degree in Telecommunication Engineering in 1998, from University of Pisa, Italy. From December 1997 to May 1998 she joined the Georgia Tech Research Institute, Atlanta, USA as a visiting research scholar where she carried on research activity in the field of radar detection in non-Gaussian background. In 1993 she joined the Dept. of Information Engineering of the University of Pisa, where she is Associate Professor since Dec. 2011. She's IEEE fellow since Jan. 2011 and she was corecipient of the 2001 IEEE Aerospace and Electronic Systems Society's Barry Carlton Award for Best Paper and recipient of the 2008 Fred Nathanson Young Engineer of the Year award for contributions to signal processing, estimation, and detection theory. In May and June 2015 she visited as invited Professor the Université Paris-Sud, CentraleSupélec, Paris, France. She has been general-chair, technical program chair and organizing committee member of many international conferences over the last 10 years. She is lead guest editor of the special issue on "Advanced Signal Processing for Radar Applications" to appear on the IEEE Journal on Special Topics of Signal Processing, December 2015, she was guest co-editor of the special issue of the Journal of the IEEE Signal Processing Society on Special Topics in Signal Processing on "Adaptive Waveform Design for Agile Sensing and Communication," published in June 2007 and lead guest editor of the special issue of International Journal of Navigation and Observation on" Modelling and Processing of Radar Signals for Earth Observation published in August 2008. She's Associate Editor of IET Proceedings - Sonar, Radar and Navigation, Editor-in-Chief of the IEEE Aerospace and Electronic Systems Magazine, member of the Editorial Board of the Springer Journal of Advances in Signal Processing (JASP), Senior Editorial board member of IEEE Journal on Selected Topics of Signal Processing (J-STSP), member of the IEEE Signal Array Processing (SAM) Technical Committees. She's also member of the IEEE AES and IEEE SP Board of Governors and Chair of the IEEE AESS Radar Panel. She's as well SP Distinguished Lecturer for the years 2014-2015, AESS Distinguished Lecturer for the years 2015-2016 and member of the IEEE Fellow Committee. Maria is a coauthor of the tutorials entitled "Radar Clutter Modeling", presented at the International Radar Conference (May 2005, Arlington, USA), "Sea and Ground Radar Clutter Modeling" presented at 2008 IEEE Radar Conference (May 2008, Rome, Italy) and at 2012 IEEE Radar Conference (May 2012, Atlanta, USA), coauthor of the tutorial "RF and digital components for highly-integrated low-power radar" presented at the same conference, of the tutorial "Recent Advances in Adaptive Radar Detection" presented at the 2014 International Radar Conference (October 2014, Lille, France) and co-author of the tutorial "High Resolution Sea and Land Clutter Modeling and analysis", presented at the 2015 IEEE International Radar Conference (May 2015, Washington DC, USA). Her general interests are in the areas of statistical signal processing, estimation and detection theory. In particular, her research interests include clutter models, spectral analysis, coherent and incoherent detection in non-Gaussian clutter, CFAR techniques, radar waveform diversity and bistatic/mustistatic active and passive radars. She co-authored many book chapters and more than 150 journal and conference papers. 